\title{
Designing for Youth-Centered Moderation and Community Governance in Minecraft
}

\author{
KATIE SALEN TEKINBAŞ and KRITHIKA JAGANNATH, University of California Irvine \\ ULRIK LYNGS, University of Oxford \\ PETR SLOVÁK, King's College London
}

Online settings have been suggested as viable sites for youth to develop social, emotional, and technical skills that can positively shape their behavior online. However, little work has been done to understand how online governance structures might support (or hinder) such learning. Using mixed-methods research, we report findings from a 2-year, in-the-wild study of 8-13 year olds on a custom multiplayer Minecraft server. The two-part study focuses on the design of youth-centered models of community governance drawn from evidence-based offline practices in the prevention and learning sciences. Preliminary results point to a set of socio-technical design approaches shaping player behavior while also supporting youth interest in Minecraftlike online environments. More broadly, the findings suggest an alternative vision of youth's capacity for ownership and control of mechanisms shaping the culture and climate of their online communities: managing player behavior while challenging current norms around adult control and surveillance of youth activity.

CCS Concepts: • Human-centered computing $\rightarrow$ Field studies; HCI design and evaluation methods; Human computer interaction (HCI); Interactive systems and tools; HCI design and evaluation methods; Field studies;

Additional Key Words and Phrases: Online Governance, community governance, rules and community behavior, community moderation and management, minecraft, social competence, positive youth development, prosocial, social and emotional learning, conflict resolution, multiplayer online games

\section{ACM Reference format:}

Katie Salen Tekinbaş, Krithika Jagannath, Ulrik Lyngs, and Petr Slovák. 2021. Designing for Youth-Centered Moderation and Community Governance in Minecraft. ACM Trans. Comput.-Hum. Interact. 28, 4, Article 24 (July 2021), 41 pages.

https://doi.org/10.1145/3450290

\section{INTRODUCTION}

The relationship between technical design and social practice has been a focus of much research in human-computer interaction (HCI) and related fields concerned with the impact of online participation on different groups and communities [20,120,158, 189]. The growing discussion

Authors' addresses: K. S. Tekinbaş and K. Jagannath, Donald Bren School of Information and Computer Sciences, University of California Irvine, 6210 Donald Bren Hall, Irvine, CA 92697-3425; emails: \{ksalen, kjaganna\}@uci.edu; U. Lyngs, Department of Computer Science, University of Oxford, Wolfson Building, Parks Road, Oxford OX1 3QD, United Kingdom; email: ulrik.lyngs@cs.ox.ac.uk; P. Slovák, King’s College London, Strand, London, WC2R 2LS, United Kingdom; email: petr.slovak@kcl.ac.uk.

Permission to make digital or hard copies of all or part of this work for personal or classroom use is granted without fee provided that copies are not made or distributed for profit or commercial advantage and that copies bear this notice and the full citation on the first page. Copyrights for components of this work owned by others than ACM must be honored. Abstracting with credit is permitted. To copy otherwise, or republish, to post on servers or to redistribute to lists, requires prior specific permission and/or a fee. Request permissions from permissions@acm.org.

(c) 2021 Association for Computing Machinery.

1073-0516/2021/07-ART24 \$15.00

https://doi.org/10.1145/3450290 
surrounding management of behavior in online spaces is tied, for example, to concerns about the impact disruptive and harmful behavior may have on children and adolescents [76, 107, 113, 196]. As Livingstone [115] and others have noted, children and adolescents are disproportionately affected by the risks of the digital world, due to their developmental vulnerabilities and their status as "early adopters" of emerging technologies [95]. With youth reporting increased time spent online-including playing games with others-parents, educators, and technology developers are eager to better understand what shapes behavior online, positive and negative behavior alike.

One promising area of research has focused on the role of community governance in influencing and managing online behavior, including conflict resolution [26, 103, 132]. Recent work by Matias [121] focused on governance in online communities too suggests the importance of social norms in regulating behavior, while research in online moderation has explored the role moderators play in guiding community behavior, working alongside-and sometimes at odds with-platform-driven algorithmic moderation [32, 71]. The digital literacy of users has a role to play in their online behavior, as well. Research from media and communication studies points to the decisive role digital skills play in helping people avoid harms and embrace opportunity associated with online participation $[75,81,112]$.

Online settings have been suggested as viable sites for youth to develop the kinds of social, emotional, and technical skills that can positively shape their behavior online [93, 94, 116]. These include skills related to social competence, the ability to build positive and healthy interpersonal relationships and to resolve interpersonal conflicts. Such positive outcomes are not automatic; however, nor should they be expected to occur in every online setting. Rather, the literature suggests that positive outcomes are much more likely to develop, if at all, within communities where young people feel a sense of agency and belonging, have friends and mentors who share an interest, and where they have access to activities that sustain their involvement $[35,64,127,156]$. Matias [121] and others, for example, have found that information about norms is influential when a person cares about a group or they feel like they belong [137]. While there is significant research on community governance, little work to date has focused on its role in supporting (or hindering) social competence outcomes in online settings, including conflict resolution. The present work aims to address that gap.

In this article, we focus on the design of a multiplayer Minecraft ${ }^{1}$ server for youth ages 8-13, highlighting the use of youth-centered moderation and community governance to foster youth interest, manage behavior, and enable personal growth. Multiplayer games are settings where youth may encounter disruptive behavior, griefing, or social drama with other players. How youth respond to such conflict relies in part, on the governance structures guiding player behavior. Work focused on the design of governance systems in multiplayer games played by youth (such as Minecraft, PopTropica, and Whyville) show governance typically reflects and reinforces structures of adult power, surveillance, and productivity [101, 147, 158, 170]. Youth are rarely asked to contribute to governance decisions and their developmental needs are often overlooked in the design of activity structures and moderation strategies. While there is a clear tension between maintaining the safety of youth online and giving them agency to act, problem-solve, and self govern, we propose that current approaches to community governance diminish their potential as sites of youth development (cf., $[89,170]$ for analogous arguments in offline settings).

\subsection{Focus of this Article}

This work, comprising a two-part study, set out to investigate how youth-centered models of community governance, including moderation and structured community norm-setting, might

\footnotetext{
${ }^{1}$ Minecraft is a popular sandbox-style video game with a multiplayer mode that allows players to interact with each other inside a Lego-like virtual world.
}

ACM Transactions on Computer-Human Interaction, Vol. 28, No. 4, Article 24. Publication date: July 2021. 
influence online player behavior in a custom Minecraft server. Study I focuses on the use of evidence-based moderation techniques from offline environments, exploring effects on engagement and agency within child-moderator dialogues. Study II extends the approach beyond moderation into the design of community-based norms and activities. We were particularly interested in exploring how evidence-based pedagogical techniques from Prevention Science ${ }^{2}$ and Positive Youth Development (PYD) ${ }^{3}$ originally developed for in-person settings [51, 109] might be transferable to an online game setting, to help address questions such as: How might community governance be designed explicitly to support youth interest, agency, and personal growth, while also maintaining their safety and security? What kinds of activity structures and moderation strategies afford youth opportunities for problem-solving and self-governance? How do youth take up and/or resist such opportunities at both individual and collective levels? Our aim was twofold: first, to examine the feasibility of using a non-authoritarian approach to online community moderation and management as a way to strengthen the capacity of participating youth to resolve interpersonal conflict on the server; and second, to extend this approach beyond moderation-only, into the design of the learning environment itself.

To start investigating this broad research agenda the work reported here takes a research through design approach [130], focusing on a particular case study context (a multiplayer Minecraft server for 8-13 year olds) and a specific aspect of personal growth (social competence). Guided by prior work in HCI (e.g., [148, 170]), Prevention Science [48, 49, 51, 68, 198], and the learning sciences [85], we have focused specifically on understanding how well-established offline social-emotional learning (SEL) and community governance models could be transferred into, and designed for, online settings. These include Community Agreements (CAs) [3, 6, 16, 52, 186, 195], reflective discussions [51]-community-created yet formalized rules that are visible to community members-and conflict resolution techniques that empower youth to problem solve and resolve disagreements on their own [87, 89, 91, 167, 169, 175]. The findings suggest the need for a closer look at social, user-driven models of online community moderation and management as tools for supporting youth personal growth and development.

\subsection{Background on Minecraft}

Minecraft is a sandbox style video game that can be played as a single-player or multiplayer experience. In the game, players can build an almost infinite number of unique creations out of cubic blocks, mining the world for raw materials and combining them to form new materials and tools. The game has two modes: Creative and Survival. Survival mode, the original game mode in Minecraft, challenges players to collect resources, build structures, battle mobs, manage hunger, and explore the world in order to survive, thrive and "complete the game" [5]. In Creative mode, players have access to unlimited resources and player avatars cannot die or be damaged by other players or creatures in the world. The game is hugely popular, especially among youth.

While Minecraft players and their creations are the most visible parts of the multiplayer game, they are supported by a class of administrators and moderators that keep each copy of the Minecraft world running. This feature of Minecraft is of interest to us here. Unlike most commercial game platforms, multiplayer Minecraft is played on "self-hosted" servers: it is players, not the game company, that manage and run their servers. They do so through a combination of pol-

\footnotetext{
${ }^{2}$ Prevention science focuses on the development of evidence-based strategies that reduce risk factors and enhance protective factors to improve the health and well-being of individuals, families, and communities. (https://www.npscoalition.org/ prevention-science).

${ }^{3} \mathrm{PYD}$ is a philosophy and approach to working with youth that emphasizes building on youths' strengths and developmental assets.
} 
icy instruments like rules and codes of conduct, moderation, and software plugins. Grimmelman [71] defines moderation as "the governance mechanisms that structure participation in a community to facilitate cooperation and prevent abuse" (p. 52). Moderators on the server act as arbiters, community managers, teachers, role models, curators, and enforcers [159] . They handle misbehavior and guide the community, both through their moderation practices and their use of plugins, which can include automated moderation tools that kick, mute, jail, or temporarily ban players [2] or grief-prevention tools that keep players from destroying other people's builds, stealing items, spamming, or chat trolling. This article focuses, in part, on moderation as a key variable shaping the culture and climate of a server, one whose design challenges more typical adult-led structures of moderation and governance.

\subsection{Overview of the Studies}

The studies presented in this article come from a 2-year, in-the-wild study of activities of players on a custom Minecraft server. In our pilot step (Study I), we aimed to understand whether introducing existing evidence-based moderation approaches to empowering youth from offline spaces could lead to similar effects online, even if all the other aspects of the online environment remained the same. To do so, we built on and extended prior work [170] by examining if-analogously to the in-person settings-less authoritarian moderation techniques (explanation and problem solving) would be associated with a higher likelihood of active engagement and increased agency from youth (reflection and constructive conflict resolution) within child-moderator dialogues. Methodologically, we approached these questions by a detailed mixed-methods analysis of 108 conflict instances sampled across 18 months of logs from the Minecraft server.

Following the promising results of Study I, the second study drew on a PYD model [42, 104, 108] to examine the possibilities of applying the "philosophy" of youth empowerment, problem solving, and self-governance to the full gameplay experience; rather than the mediation process only. Our aim was to (i) re-design the online environment to remove common technical "solutions" to interpersonal conflicts, ${ }^{4}$ (ii) replace these with youth-led community norms (called CAs) which were facilitated through pedagogical structures from offline settings, and (iii) design game activities to maximize opportunities for youth to reflect and iterate on formalized community norms, in a safe environment. For this article, our analysis of Study II is focused on understanding the various ways in which youth participated in establishing, negotiating, revising, and reinforcing the community norms of the program over a 6 month period. Our qualitative findings suggest that CAs gave youth agency over the culture and climate of the online environment, and were used by youth to express interests and reinforce social connections, while also addressing safety concerns.

\subsection{Summary and Contributions}

While only a first step toward answering the broad research agenda set out above, the preliminary findings presented here point to a set of socio-technical design approaches to youth-centered moderation and community governance that fosters youth interest, manages behavior, and supports personal growth in Minecraft-like online environments. More broadly, the studies offer a model of young people's capacity for ownership and control of the mechanisms shaping the culture and climate of their online communities. Our findings do not suggest that adults have no role to play in managing player behavior; some situations necessitate adult intervention. Rather, their role rests in helping youth develop productive mindsets around their behavior, and in helping them develop the ability to problem solve with others. Moderators have a key role to play in

\footnotetext{
${ }^{4}$ Such as preventing stealing by introducing "claims" command in-game, fights by disallowing player-vs-player combat...[170].
} 
guiding youth through such experiences. Existing work from the prevention sciences and other fields suggests that-at least offline-experience in problem-solving and engaging with situations of conflict and its resolution is foundational for the development of social competence [51, 82, $144,153,191]$. While future work will need to verify the extent to which online environments are effective in facilitating lasting development of such skills (and whether these can transfer to out-of-game contexts), the work here is promising.

\section{REVIEW OF RELATED WORK}

The social dynamics of online communities, including games, have been explored from numerous angles $[77,122,158,159,180]$. In this work, we focus on a game server where youth meet to play, share interests, socialize, and build a community [129], emphasizing the social nature of the server and the interactions between players and moderators. While much work has been done in recent years to understand the digital media practices of youth-including research focused on learning and motivation, interest, identity, social development, and creative expression $[27,114,116,126$, $127,136]$-little work is available on the impact of online governance on the social competence of young players [170]. Similarly little work focuses on the design affordances of online learning environments for youth, including games, that support youth development. In the following sections, we first introduce existing work in the learning and prevention sciences related to connections between learning, shared interest, and community. We go on to present a snapshot of HCI scholarship related to online governance including literature on rules, norms, and community development, before taking a particular look at models developed for offline environments, like classrooms and afterschool programs, which emphasize youth development. We then move on to a specific discussion of how social learning can arise as part of participation and interaction with the governance structures of online games. We use this literature to help frame the ongoing community governance work by youth in the program of study, highlighting the ways youth use agreements to express both individual and collective values, and negotiate the norms of their community.

\subsection{Learning, Shared Interest, and Community}

The period from around 12 to 18 years is a critical time when individuals form interests and social identities [27, 33, 85, 156]. Research on development emphasizes the importance of participationchoice and voice-for adolescents [38, 74, 138]. According to Pittman, development is triggered by engagement-"young people learn best when they are engaged with their heads and their hearts, and where they have real choice in the situations in which they are involved" [140]. Further, the learning sciences have increasingly recognized the role culture, social relationships, and shared practice play in the discovery of interests and persistence in pursuing them [38]. Research also documents how the availability of shared activities [11, 12], familial support [41], and rapport with teachers and mentors [181] matter far more than formal instruction in the development of scientific interests. Damon and others [28, 43, 85, 119] further emphasize that a central indicator of youth thriving is engagement in pursuits that serve the common welfare and make meaningful contributions to communities [110]. Youth go beyond their own self-centered needs and extend outward to the pursuit of goals that benefit the world.

Decades of research in educational and positive psychology in offline settings, including schools and after-school programs, emphasize the role of community, agency, and interest of the learner as key enablers of personal growth $[85,104,105,150,151,194]$. However, we are still in the early stages of bringing this research into the design of digital environments, and games more specifically. Recent research into games that promote prosocial behavior [54, 143, 187, 188], learning [57, 60, $65,157,176,177]$, and healthy behavioral changes [13] suggest that digital games do have the 
potential to encourage healthy social outcomes and youth well-being. Yet more work needs to be done from both an educational technology and $\mathrm{HCI}$ perspective to understand how to design for these desired outcomes.

\subsection{Rules, Norms, and Community Development}

Existing literature on online community moderation suggests that communities evolve over time, "as a result of rule-breaking, rule-making, and rule-enforcement" [179]. As rules and norms develop and change, often in response to short-term events or transitions, they affect moderation decisions. These decisions, according to Seering [159] and others who study platforms like Twitch, Reddit, and Facebook, are most often made by moderators, without input from general community members. In contrast, recent research on youth participation in online affinity networks-networks organized around an interest in gaming, political organizing, fan fiction, or hip hop, for examplehighlights community-based models of moderation and self-governance [18, 58, 86, 97]. Effective networks build in ways for participants to take ownership of community values and model them for others, giving youth agency over their environment, addressing safety concerns, and ensuring that the values and norms remain culturally relevant and resilient [59, 86]. In effect, participants in such networks share an interest, a culture, and a purpose; and it is the combination of values, norms, and practices that makes the culture of each network unique, drawing on the cultural knowledge, prior experiences, frames of reference, and performance styles of its participants [85]. The agency afforded through such a process has been shown to provide a strong foundation for SEL and personal growth [86]. The present work aims to contribute to the literature on rules, norms, and community development in online settings through a focus on governance structures that support youth development while also managing online behavior.

\subsection{Approaches to Supporting Youth Development}

Progressive educators, juvenile justice advocates, psychologists, caregivers, and others have developed non-authoritative and child-centered approaches to youth development, whereby youth are supported in developing social, ethical, emotional, physical, and cognitive competencies. These approaches enhance protective factors in youth and build competencies that enable more positive interactions with their environment, "...leading to healthier development across developmental domains (i.e. areas of development such as cognition, social/emotional and language/communication)" [56]. We draw on several of these approaches in our work, including PYD [104, 160-162], healthy youth development [139], and SEL [133, 183, 192]. We do so with the specific aim of increasing understanding of how such models, which have been developed and used primarily in offline settings, might be effectively integrated into online settings in ways that leverage the particular affordances of multiplayer game environments.

2.3.1 Positive Youth Development. PYD is an approach that advocates for providing youth with contexts and opportunities tied to their interests and focused on their strengths as opposed to their shortcomings [42, 72, 104, 141, 154]. PYD has been used in both formal and informal learning settings like schools and after-school programs over the past 20 years, with promising results [21, 50, 63, 67, 96, 108, 138, 160]. Studies of offline PYD programs highlight approaches to promoting positive development [30,31, 110] but offer little information on how offline PYD interventions specifically track contexts and activities that youth partake in [104]. Further, there is a lack of understanding of how features of offline PYD programs might be adapted to an online context. Within HCI there has been no work that we know of focused on approaches to PYD in the context of online communities. The preliminary results presented here begin to suggest some potential and much needed approaches. 
2.3.2 Social and Emotional Learning. Another line of work-which has yet to be examined in depth by HCI researchers (cf., [173])-originates in the field of Prevention Science with programs developed for both at-risk and population-wide communities, often in view of improving students well-being and resilience competencies (e.g., [66, 68]). These formal programs are predominantly deployed through schools and target, for example, personal growth such as increases in social competence, emotion regulation, or problem solving; they are often deployed as part of violence prevention frameworks. A substantive body of research provides evidence for feasibility and effectiveness of such interventions in youth populations in developing key cognitive and emotional competencies that reduce the incidence of developing behavioral problems in later life (e.g., [8, 190]). In the context of this research, some of the most relevant programs include Resolving Conflict Creatively [7, 44], Peacemakers [88], the 4Rs Program [1, 90], and I Can Problem Solve (ICPS) [164, 167-169, 175], which had been implemented in offline settings like schools, to help adolescents develop conflict-resolution and problem-solving skills. To best of our knowledge, however, only very limited work has been done so far to investigate if/how these programs could be adapted for use in online settings (cf., $[171,178]$ ), with recent work suggesting the plausibility and potential efficacy of such a transfer [170].

\subsection{Game Communities and Social Learning}

In order to understand how large is the potential that a medium has to change an individual, researchers have typically measured how realistically a user behaves while inside of that medium [80]. In this work, the mediated behavior of individuals (behavior within an online world, for example) compares to actual behavior. Yee and others [197] have shown that social behavior and norms in virtual environments are comparable to those in the physical world, suggesting that online games can be used as valid platforms to study-and perhaps impact-social interaction. Online play-based communities mediate social experiences for youth $[36,148]$ and offer authentic contexts in which to develop SEL skills, including skills related to conflict resolution and problem-solving [170]. Such experiences are linked to apprenticeship within communities of practice [59, 105, 193] that emerge as part of the social interactions around the game-such as when new players are "shown the ropes" by other community members. Through such cognitive apprenticeship [98, 99, 184], players are "enculturated" into both the technical practices of the game, as well as the dominant cultural perspectives: "this includes adopting the 'right' set of values and attitudes toward the game, its content, its goals, world, and other players" [60, p. 123]. Previous work has examined how these processes might impact gamers' perspectives toward collaborative problem-solving, digital media practices, computational literacy as well as social dynamics in online games [45-47, 70, 89]. Relatively few studies, however, have built on this research to explore how to explicitly design online game settings to support the development of social competence in players. In Beanstalk [34] and ZooU [39], for example, researchers used the design of in-game characters to examine children's ability to self-regulate and persist over challenges presented by the games. Designing for social interactions among players was not part of either study. This leaves open the question of how we might design SEL interventions that enable youth to socially interact with peers and other adults to grow social competence. Our work addresses this gap by providing young players (8-13 year olds) and their young adult educators (18-24 year olds) with access to a moderated online community in Minecraft where conflict resolution and prosocial behavioral norms are supported, encouraged, and modeled.

\subsection{Current Approaches to Moderation in Online Gaming Communities ( $\mathrm{HCI})$}

As game communities continue to grow in size and complexity, so have the challenges around online moderation, which are well documented in the HCI literature and elsewhere $[10,55,102$, 
174]. Current models of human moderation are expensive and inconsistent at scale and automated approaches currently lack the sophistication needed to support a diverse community of players. Governance is typically top-down, autocratic, and punitive [4]. A uniting feature of the existing strategies to manage player behavior online is the reliance on technological deterrence approaches $[17,19,101,118,158,159,170]$ : the threat of bans or other in-game punishments for infractions, which can be based on crowd-sourced decisions by other players (e.g., the "Tribunal" in League of Legends [117] or machine learning approaches [100,101].

A similar deterrence-based approach to moderation is also present in games and virtual worlds specifically designed for tweens-such as WebKinz, Neopets, Club Penguin, Whyville and otherswhere stable social bonds and on-going relationships among peers are among the key features [23, 84, 85, 92, 155]. Work by Ringland et al. [148] uncovers a similar dynamic of strict rules and technological restrictions has played out also in more specialized servers, in this case a Minecraft community supporting children with autism; and Slovak et al. [170] surface similar authoritarian approaches also in the after-school Minecraft server that serves as a case for the work here. However, the direct impacts of such approaches on child learning or engagement with conflict have not been investigated so far. The widespread use of authoritarian techniques by software developers, educational technologists, and others has been also likely encouraged by parental fear and anxiety around screen time, online predators, and potential for cyber-bullying within multiplayer games (cf $[146,148])$. While these interventions effectively regulate the worst behavior that could emerge in online spaces, they do so at a cost of limiting learning opportunities for youth $[78,79$, 170]; further, such pedagogical techniques have long-been discarded by progressive educators in analogous situations offline (cf., 2.1 and 2.2 here).

In summary, while there is a growing body of literature on governance and moderation in online games, little of this research has focused on the developmental needs of youth. Similarly, while we have an abundant literature on alternative mediation practices in offline settings, drawing on Prevention Science literature (e.g., ICPS [169]) and models for promoting PYD (e.g., [104]), next to no work exists that would investigate whether and how these offline practices can be adapted for online settings to produce similar outcomes-building social, emotional, problem-solving and conflict resolution skills in youth. The research presented in this article aims to start addressing this gap while also offering a 21st century model for online teaching and learning that places youth interest and agency at the center.

\section{STUDY I: EFFECTS OF MODERATING STYLES IN MINECRAFT}

The goal of the first study was to examine the effects of moderation styles on child responses and engagement in conflict resolution within a case-study Minecraft server setting. This aim was motivated both by the broader gaps identified above, as well as a prior study [170] emphasizing the (potentially unintended) disempowering effects of adult-driven and deterrence-based moderation approaches in online games. By focusing on the associations of child responses with different moderating styles from historical log data, the observational study methodology served as a first step in understanding any potential power imbalance that might prevent youth from engaging more actively in the conflict resolution and server administration; as well as point to plausible changes in the moderating approach to alter such disempowering effects (drawing on the Prevention Science literature described below).

Theoretical Grounding: Theoretically, the observational methodology was grounded in a wellestablished model of social competence development in offline settings: Interpersonal Problem Solving (IPS) [37, 153, 169]. We expected that this general approach would likely fit well with the social affordances of online game play, as it is theoretically grounded in the effects of external 
mediation on agency within interpersonal conflicts. Specifically, we drew on the "I Can Problem Solve" program by Shure and Spivak $[168,169]$ as a particular instance of the IPS pedagogical model, for two reasons. First, like all IPS programs, IPS uses cognitive-behavioral training to develop youth's problem solving-focusing on helping youth develop models for how to think, rather than what exactly to think [123, 164-169]. This makes the underlying theory less reliant on teaching exact behavioral skills (such as eye-contact, wording, or tone of voice); the approach therefore seemed promising for use within online contexts. Second, the key active learning component of ICPS programs is "dialoguing" [167, 168]: a conversational mechanism by which a mentor-usually a teacher or a parent-scaffolds child thinking about a particular problem through carefully selected questions.

Existing Research Gaps: Prior work has indicated that such dialoguing approaches could be, theoretically, incorporated into the in-game moderation practice, without requiring formal "lessons" for the players $[170,172]$. An important benefit of this approach would be the possibility to embed an intervention into the social-fabric of the gaming environment in order to leverage natural, authentically arising situations for the nurturing of social competence. If the conversational mechanisms used to facilitate youth problem solving techniques in existing (offline) programs are shown to facilitate similar effects in-situ online, the research community can draw on the existing knowledge from offline contexts to also utilize the well-established intervention mechanisms in a range of prospective online interventional approaches. Developing an initial understanding of transferability of these mechanisms into online settings is the key research aim of Study I. Specifically, if we were to see more active engagement of children depending on online moderation practice in a Minecraft server-analogous to what has been observed in offline setting-this would provide a crucial piece of evidence to support the potential (and a well-established evidence-base) for empowering, community driven design mechanisms argued for in this article.

\subsection{Case Study Context and RQs}

As part of the observational study, we collected a number of moderator-child chat interactions in which moderators mediated a resolution for a particular conflict that had arisen on the serverwe will refer to these chat snippets as "conflict mediation logs." The primary research aim of this study was to then investigate whether differences in mediation approaches from moderators would be accompanied by corresponding differences in youth responses/behavior,as based on ICPS theory. To do so, our analysis focused on a particular online after-school setting called "Kid Club" (KC): a moderated Minecraft server for youth. Activities on the server supported unstructured, collaborative play organized around youth interests. KC data were selected as a proof-of-concept context for several reasons: (1) the persistent nature of the environment (e.g., player resources were maintained across game sessions), together with unstructured game play, led to the potential for high conflict rates; (2) all communication between players happened over the chat channels (no voice communication was available) and the players were connecting from different physical locations, which allowed us to capture all interaction between players through the server logs; and finally (3) KC ran continuously for the full length of data collection, allowing the team to sample from multiple time points to explore temporal stability of the observations.

As part of a larger project, the research team had access to 18 months of logs from a Minecraft server used by youth aged 8-13. We sampled 108 conflict interactions out of this broader dataset, sampling across two time periods. Each conflict instance was then divided into individual in-game utterances and coded, with the coding procedure theoretically grounded within the ICPS typology. This led to altogether 1,511 coded utterances, with 693 originating from youth, and 818 from moderators. The coding scheme for youth responses emerged initially from thematic analysis (leading to a fixed code book-see Appendix A.2.3). The Cohen's kappa scores for the final coding scheme 
Table 1. Distribution of Analyzed Utterances across Youth and Moderators within The Observed Periods

\begin{tabular}{lccccc}
\hline $\begin{array}{l}\text { Observation } \\
\text { Period }\end{array}$ & \#Conflicts & \#Youth & \#Moderators & $\begin{array}{c}\text { \#Youth } \\
\text { Utterances }\end{array}$ & $\begin{array}{c}\text { \#Moderator } \\
\text { Utterances }\end{array}$ \\
\hline Summer'15 & 32 & 21 & 16 & 231 & 273 \\
Dec'16-Feb'17 & 76 & 22 & 17 & 462 & 545 \\
\hline
\end{tabular}

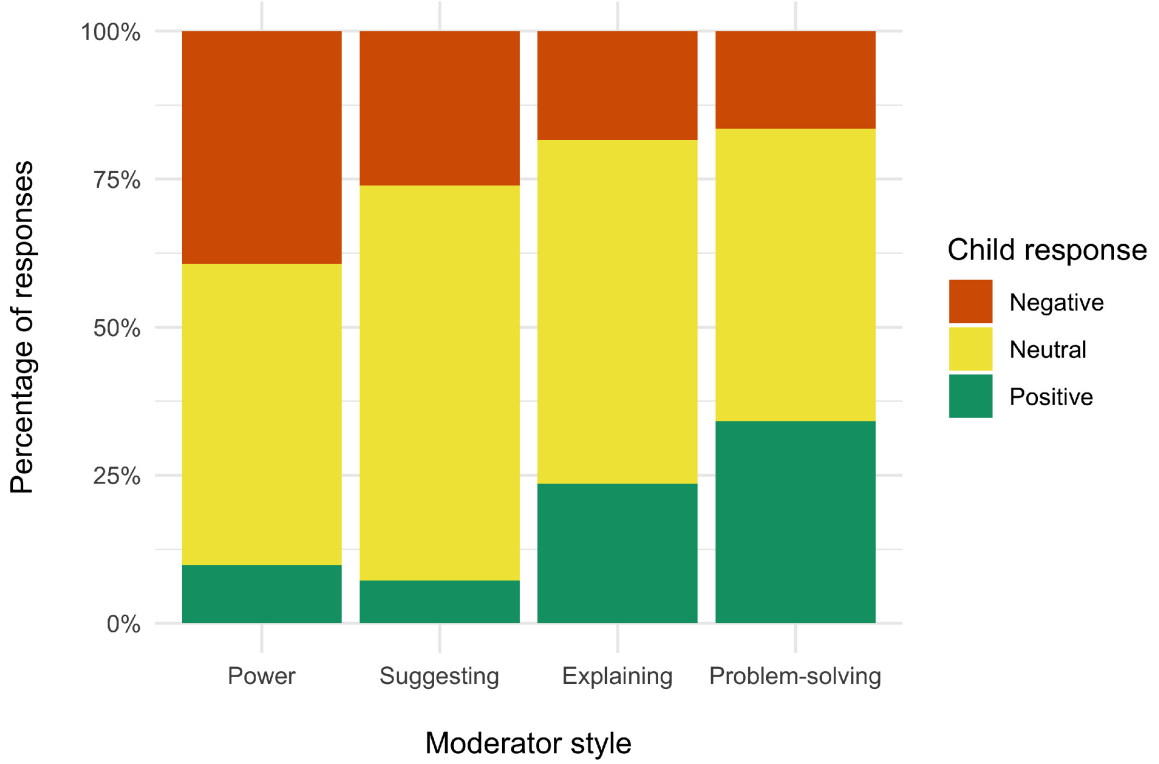

Fig. 1. Valence of youth responses to moderator utterance in different styles in conflicts on a moderated Minecraft server across periods (a).

and $\%$ agreement were kappa $=0.965 ; \%$ agree $=97.1$. The Appendix A provides more information on the data collection approach and methods used.

\subsection{Study I Results}

The aim was to explore whether these naturalistic moderator-youth interactions would show consistent patterns depending on mediation style, as expected from the (offline) Prevention Science literature. Specifically, we explored whether-analogous to use in-person settings-the less authoritarian mediation techniques (explanation and problem solving) would be correlated with a higher likelihood of active engagement and increased agency from the youth (reflection and constructive conflict resolution). If so, this would suggest the benefits of re-framing moderation approaches toward the dialoguing and problem-solving methods outlined above. In what follows, we first provide a short descriptive summary of the dataset, present the quantitative analysis comparing the patterns of moderator-youth interaction, and then illustrate the quantitative observation on a qualitative example, to showcase the impact of the different moderating approaches on the server.

3.2.1 Descriptive Analytics. Out of 1,522 coded utterances, we first dropped utterances in which youth had communicated with other youth, leaving us with a final dataset of 1,511 utterances. Table 1 shows the numbers of utterances by youth and moderators within the observation periods. 




Fig. 2. Valence of youth responses to moderator utterance in different styles in conflicts on a moderated Minecraft server between periods (b).

3.2.2 Primary Results. A Chi-Square test showed a significant association between the type of moderator style and the valence of youth's subsequent responses, $\chi^{2}=39.55, p<0.001$. Compared to a power-oriented style, the odds of getting a positive rather than negative response were 8.29 times higher when the moderators used problem-solving utterances, and 5.12 times higher when explaining. Figure 1 visualizes these differences in youth responses associated with the range of moderating styles. A simple moderator analysis showed that the differences in distribution of valence of youth utterances in response to different moderator styles were similar in both sampled time periods, as shown in Figure 2. The association between moderator style and the valence of youth's responses was statistically significant in both observation periods (Summer'15: $\chi^{2}=17.43$, $\mathrm{p}<0.008$; Dec'16-Feb'17: $\chi^{2}=25.79, \mathrm{p}<0.001$ ). The in-situ naturalistic collection across two time periods, separated by approximately 18 months, makes any controlled analysis of effects of individual moderators or children difficult, as only a few were present across both periods. Overall, these quantitative results suggest a pattern of responding that is analogous to offline interactions: youth appear to consistently differ in their responses to the four classes of mediation, with Problem Solving and Explanation associated with highest ratio of positive responses from youth, i.e., responses that were either classed as reflective or directly restorative (making up with the other person). To illustrate how such differences manifested in the actual conversations, the following section outlines two exemplary excerpts.

3.2.3 Example Conflicts. While full qualitative analysis of the coded dataset is beyond the scope of this article, it is useful to illustrate the striking qualitative differences we observed across a sample of excerpts, selected as exemplary instances of the common patterns we saw across the dataset. As such, these can be read as a qualitative illustration of the types of effects shown in quantitative data outlined above. In the interest of length, we selected an example of a "power" mediation, as the most commonly used approach (cf., [172]); and "problem solving" as the target approach adapted from Prevention Science. The illustrative examples for each presented herein come directly from the raw data, with no edits apart from removing some interim lines for length and anonymizing the Minecraft usernames. 
Table 2. Exemplar for Power Mediation Approach: Participant Appears to be Inured to Punishment/Ban

\begin{tabular}{lc}
\hline Power Mediation Exemplar & Applied Codes \\
\hline Mod2: You know I still remembered what you did P2. It's not cool & power \\
to skip out and pretend nothing ever happened &
\end{tabular}

P2: if your going to be like this then maybe a will just leave!

negative, uncooperative

Mod2: That's fine, you can feel free to leave.I'm just going to let power you know when you signed up for this server, you agreed to our rules. We gave you multiple warnings and were willing to talk it out with you but you really disappointed us when you ran away and ditched us

P2: do you think anyone else will come in the server soon besides mods

Mod2: I'm going to give you one actual final warning P2, any issues today and we're putting you in cooldown for a talk

P2: k. i'll go play spleef with myself Mod2: Okay then, you do that

negative, uncooperative power

P2: brake 5 block's then jump in the hole that's how you play spleef negative, uncooperative forver alone

- In the power mediation example shown in Table 2, the moderator (Mod2) is polite, but clearly addresses the youth (P2) with a hierarchical mindset. The focus of the moderation is on P2's past behavior (rather than on positive next steps), resulting in a reduction of the youth's agency to expectations of admitting fault and repentance. P2 remains oppositional throughout the dialogue, reduces their engagement with the moderator, and appears indifferent to punishment. This response is consistent both with the reactions to power-like approaches used in offline settings (cf., [164]), as well as the limited work in online settings (cf., [170]).

-In contrast, Table 3 offers an example of a problem-solving dialoguing approach to moderation. The moderator starts by referring to past behavior of the youth (P3), but instead of making this the focus, as in the previous example, it is used to provide space for the player to explain the situation. The moderator then follows with a line of non-judgemental questioning, which allows P3 to reflect on what has happened, respond to and validate P3's feelings ("I agree P6 didn't handle it well"), while also keeping the conversation focused on P6's behavior and his/her thoughts on how similar situations could/should be handled. In response, P6 appears more engaged and reflective in the conversation, and is given agency to explain and make choices for the future. While the power interaction could be read as two people "speaking over" each other, the conversation flow here is much tighter and responsive; potentially leading to higher engagements. No threats are used (or apparently necessary) and social norms and server rules are referred to indirectly ("... do you think it was a good thing to say to him?'). Again, such patterns are consistent with existing ICPS literature and illustrate the substantial differences in positive/negative odds uncovered by the quantitative analysis. 
Table 3. Exemplar for Problem Solving Approach: Youth Is Reflective About Their Emotional State and Behavior

Problem Solving Mediation Exemplar

Mod3: P3 Remember the conversation we had about how you treat other players the other day? You're just a SAD NOOB!" are your words from today

P3: He was saying stuff that made me uncomfortable."You're a LIER" But yeah, what I said was not a good response

Mod3: Why did he call you a liar?

P3: Because I said he was a bow spammer ;-;

P3: Which is true,he was spamming arrows at me

Mod3: Might have been true. Do you think it was a good thing to say to him?

P3: No, not really.But I feel that he was being a little rude to me too.

Mod3: I can see the whole log. I agree P6 didn't handle it well. But that doesn't have much to do with how you handled it

P3: I know :(

Mod3: P3 take a minute and re-read what you type before you send it. If you wouldn't want someone to say it to you don't say it! positive, reflection

problem solving

positive, reflection

neutral, explaining

problem solving

positive, reflection

explaining

positive, reflection

suggesting

\subsection{Study I Discussion}

The pilot results outlined in the section above are promising: the quantitative findings suggest that there are substantial effects of differing moderator styles on youth responses; and that these findings appear consistent across two different time periods (and thus, to a large extent, also likely present across different youth and moderator samples). The observed correlations of positive versus negative youth responses with different moderating strategies were also consistent with the ICPS theoretical predictions from offline contexts, suggesting that language and framing of conflict mediation within an online setting could have similar effects as those observed within in-person contexts. These quantitative findings were supported by qualitative observations, as illustrated by the log example interactions. Especially in the relatively rare instances when moderators applied problem solving moderation consistently, the interactions seemed to lead to a substantially different youth behavior and engagement in the conflict's resolution (see also Study II here). When combined with prior work (e.g., [170]), these results support the possibility of developing youth centered, agency empowering mediation approaches, while drawing on mechanisms from offline Prevention Science programs.

As with every observational study, one inherent limitation of the design is the availability of correlational findings. As such, we are not attempting to claim any causal effects of moderating strategies on youth responses; although the qualitative differences we observed seemed quite persuasive. This is partially mitigated by the ability to contrast any found patterns online with the 
existing body of evidence in offline settings, which includes Randomized Control Trial or RCTbased interventional settings. The second key limitation is drawing on in-situ data from a "live" server, which leaves the researchers with no control over the types of mediation sampled, or the ratios across moderators and youth (e.g., some youth and moderators might be engaged in many more conflicts than others). Our design has however tried to balance the sampling across the mediation situations that have happened on the server, thus reflecting the structural differences in conflict engagement rates and moderation approaches among moderators/youth in the online setting. An associated issue was that the data was collected over time within an on-going community, so it is likely that individual conflict instances are not strictly independent: previous interactions for particular mod/youth pairs might have had trickle down effects to later conflict mediation instances.

However, the in-situ data collection also constitutes a strength of the research methodology: sampling widely across conflicts appearing on a "live" server strengthens the ecological validity of the findings, and any consistent patterns found might be more likely to translate into real-world impact. Moreover, the strong theoretical grounding in empirical Prevention Science intervention data from offline interactions provides a good explanatory framework for the observed effects, as well as point of contrast. The General Discussion section will further discuss the Study I findings, connecting these with the results of Study II, data which we turn to right now.

\section{STUDY II: DESIGNING FOR YOUTH-LED COMMUNITY GOVERNANCE IN MINECRAFT}

Data from Study I reveals that youth responded positively when they were supported in resolving interpersonal conflicts on the server. An ICPS approach to moderation created a context in which youth were shown to be more insightful and resourceful in solving the problems they encountered. However, we saw during the study that these characteristics (i.e., being insightful, reflective) emerged mostly only after youth were prompted by moderators, i.e., only after the conflict seemed significant enough to warrant a moderator involvement.

In the next phase of work, we became interested in understanding how to shift the responsibility for engagement in reflection and problem-solving into the hands of youth themselves. We used the process by which youth formed and reformed CAs as a touchstone for understanding the complex interplay between design choices, social practice, and values shaping player experience of conflict and community on the server. How might strategies underlying a non-authoritarian approach to moderation be applied to the design of the Minecraft server environment, for example, as a way to manage player behavior on the server? Which social and technical features could be leveraged to increase opportunities for youth to engage in productive conflict while also maintaining their safety and security? In what ways might ownership over the creation, negotiation, and enforcement of community norms reflect their values and needs?

To answer these questions, we developed and piloted an online after-school program on a multiplayer Minecraft server; we called the program "Survival Lab." In the following sections, we focus specifically on an analysis of the CAs created and held by youth participants in Survival Lab (See Appendix B.2) as a key component of a youth-centered governance model designed to support youth personal growth and development.

\subsection{Program Design Considerations}

Drawing on related research that highlights choice, voice, ownership of community values, and opportunities to model them for others as key variables supporting youth agency and engagement, we established a set of fundamental design principles to guide the development of Survival Lab. First, we aimed to remove any of the "technical solutions" that are traditionally used on Minecraft 
servers and similar youth-oriented virtual environments to preclude disagreements between participants. The traditional design blueprint is to identify the kinds of situations where conflict is likely to arise between players, and then remove the possibility of such interaction from the game: if conflicts commonly arise as players attack each other, the solution is to remove the ability to attack other players; if players tend to destroy or modify others' buildings without permission, the solution is to introduce in-game claims, where only pre-specified players can interact with the blocks in each region; if players steal from each other, the solution is to introduce "lockable" chests, and so on.

Second, we deliberately designed the in-game events and world structure in ways that maximized the need for players' cooperation and interdependence, such as resource scarcity or goals that had to be met collectively. This approach used shared activities as the backbone for social learning, providing youth with opportunities for self-expression, choice, and contribution, as well as a chance to collaborate, cooperate, and compete (e.g., [14, 22, 69]). Shared activities created the potential for interpersonal conflict, as youth were challenged to co-plan, negotiate priorities, and make choices focused on collective outcomes; they did so, however, within a collaborative framing where disagreements could be framed as a "teachable moments" for the community as a whole, as exemplified in Section 4.3.1.

As in Study I, we introduced evidence-based youth development techniques from offline settings that-we hoped-would serve to replace traditional externalized moderation with self-directed social norms. Specifically, we drew on two main evidence-based social and emotional learning strategies from offline programs in schools: youth-authored CAs and a set of opening and closing discussion "rituals" that provided recurring opportunities for the group to reflect and discuss recent events. These reflective rituals helped youth to actively engage in self-governance by providing a consistent opportunity for them to consider their needs and values in the context of communitybuilding.

Finally, we established a clear role and expectations for moderators on the server. Moderators facilitated both the CAs work with youth, and created the prompts that formed the basis of the reflective rituals. Building on the approach piloted in Study I, moderators were expected to use the "problem-solving" approach if any mediation was needed; and the key notion of scaffolding youth thinking (rather than providing solutions) was also expected to underpin any moderator-youth interactions, such as those during CA creation or opening/closing rituals. This combination of an "easing" of technical controls in favor of player-led resolution, an emphasis on cooperative and interdependent play, supportive moderators, and the integration of problem-solving scaffolding and rituals informed the program's design at all levels.

4.1.1 Descriptive Analytics. We designed Survival Lab using Minecraft's Survival mode, which enabled the online moderators to create in-game quests and other activities based on game mechanics like limited resources, mobs ${ }^{5}$ with the power to kill player characters, and an in-game economy. Survival Lab ran as a drop-in program for 90 minutes twice a week, for 60-months (72 hours total). College students served as server-world designers and online moderators trained in the tenants of the ICPS model highlighted in Study I. Table 4 outlines key program features. Figure 3 shows the program's structural features.

\subsection{Methods and Data Collection}

Findings emerged from a combination of trace ethnography techniques [61] and recording of ingame observations [185]. Trace ethnography has been used in similar online play-based settings

\footnotetext{
${ }^{5}$ Mobs in Minecraft are living creatures that move around in the game. Mobs can be passive, neutral, or hostile.
} 
Table 4. Survival Lab's Key Program Features

\begin{tabular}{ll}
\hline Program Feature & \multicolumn{1}{c}{ Description } \\
\hline CAs & $\begin{array}{l}\text { Expectations that members of a group or community create } \\
\text { together and agree to follow, as a way to guide respectful and } \\
\text { effective participation. CAs are flexible: youth are encouraged to } \\
\text { discuss and update the agreements as needed. Youth are responsible } \\
\text { for the creation of the CAs without input from the moderators, } \\
\text { beyond being encouraged to phrase the agreements positively }\end{array}$ \\
Opening and closing & $\begin{array}{l}\text { Moderated group discussions tied to events and interactions that } \\
\text { rituals } \\
\text { to reflect on community issues, make changes to CAs, and celebrate } \\
\text { their play }\end{array}$ \\
Communication tools & $\begin{array}{l}\text { Youth communicate through Minecraft's text chat system, and with } \\
\text { TeamSpeak }\end{array}$ \\
Play challenges & $\begin{array}{l}\text { Time-bound challenges and crisis events within the Minecraft } \\
\text { world. Challenges are designed to maximize conflict between } \\
\text { players and groups of players as a means of cultivating } \\
\text { problem-solving, collaboration, and conflict resolution skills }\end{array}$ \\
\hline
\end{tabular}

a. 90 minute session structure

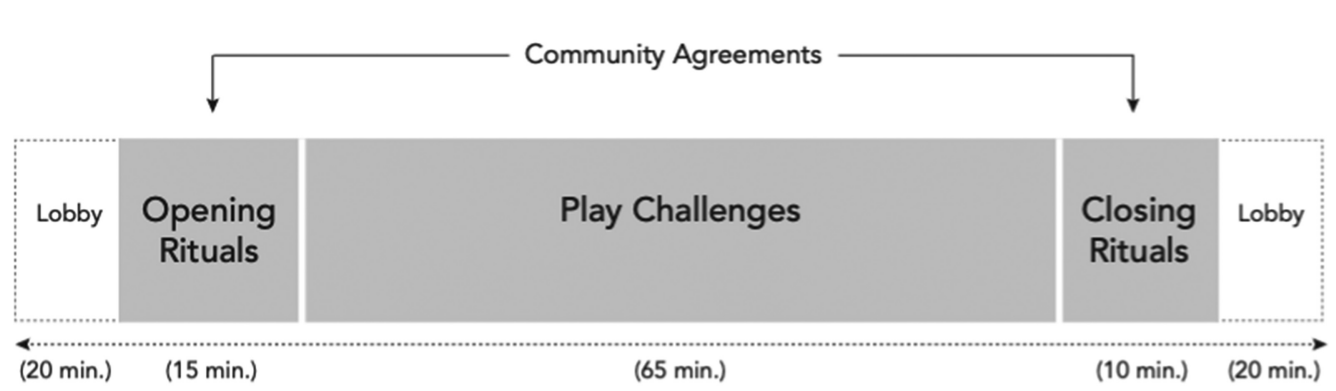

b. Teamspeak structure

\begin{tabular}{|c|c|c|c|c|}
\hline $\begin{array}{c}\text { Main } \\
\text { channel }\end{array}$ & $\begin{array}{l}\text { Main } \\
\text { channel }\end{array}$ & $\begin{array}{c}\text { Youth free to go into team-specific channels } \\
\text { or remain in the main channel }\end{array}$ & $\begin{array}{l}\text { Main } \\
\text { channel }\end{array}$ & $\begin{array}{c}\text { Main } \\
\text { channel }\end{array}$ \\
\hline
\end{tabular}

Fig. 3. Survival Lab’s structural features.

[142] and is useful in corroborating in-game observations, as it offers documentary data generated by the online environment to validate qualitative observations [73]. Online social settings, similar to offline social environments, involve layers of activity between participants that unfold in realtime and virtual space. While traditional participant-observation and ethnographic methodologies within such online settings enable gathering select participants' data, these techniques are limited in that they capture information related to a given participant in a specific point in time and virtual 
space (e.g., [106]). Traces of digital data including video, audio, and text logs that are automatically generated by socio-technical systems, however, can help researchers to re-construct information and obtain additional perspectives that mimic the real-time and virtual space within which online social interactions take place [106]. Traces therefore allow for a more granular description of how participants created and revised CAs, how CAs changed over time, and what kinds of activities and interactions informed revisions to CAs.

4.2.1 Dataset Used in this Work. We collected 100 hours of in-game observations between September 2018 and January 2019, as well as log data including video replays from the researcher's and each of the four moderator's perspectives, audio recordings of various Teamspeak channels (amounting to nearly 5,000 hours of log data), screenshots, Minecraft text chat logs, moderator logs from Teamspeak and Slack, and planning documents. The research team participated in Minecraft in spectator mode, which allowed them to fly around and observe the world without interacting with it, or participants, in any way. Researchers were visible to youth participants on TeamSpeak. While youth were aware that researchers were observing the program they were not aware of their research objectives or the program's underlying pedagogical goals.

4.2.2 Data Analysis Process. Using purposive sampling (e.g., [40, 62, 149]), we inductively analyzed the observational data, traces data, field notes, and analytical memos to identify a series of salient examples to understand how participants engaged with the program. Specifically, we began by listing all instances of youth interactions with three of the program's key design features: CAs, opening and closing discussion rituals, and team-based challenges (135 instances, 85 hours of observational data, and additional 350 hours of video and audio traces data). We built a dataset with partially transcribed data, field notes, and analytical memos using cloud-based software and spreadsheets. We revisited the traces audio and video logs, as often as needed, to iteratively construct richer descriptions of participants' interactions with these design features. We used the criteria of time (i.e., at what stage in the program the interaction occurred, such as Week 1 and Week 14) and activity (i.e., what participants did and how they communicated, via text and/or voice utterances) to identify instances when participants engaged with the CAs, opening and closing discussion rituals, and team-based challenges. For each interaction, we compared the observational notes against the traces data including video and audio logs.

4.2.3 Participants and Ethics. A total of 30 youth, ages 8-13 years, participated in the program, with different degrees of frequency. A total of 10-15 participants typically participated in a given session. Participants' level of experience with the game varied, with all participants having at least some knowledge of the game. Four moderators (Mod1, Mod2, Mod3, and Mod4; ages 18-24 years) facilitated the sessions. Additionally, one community ambassador (CA1, ${ }^{7} 15$ years) attended. The study was approved in accordance with the rules and regulations stated by the ethics committee within the Institutional Review Board at the US university. Parents of all participants granted permission for observations and access to server logs as part of the program registration process. Researchers did not have permission to interact directly with youth participants; moderators and community ambassadors gave informed consent prior to the study.

\subsection{Study II Results}

The primary research focus of Study II was to examine outcomes related to the pairing of youth-led governance (CAs) with the release of technical solutions limiting interpersonal conflict, and ac-

\footnotetext{
${ }^{6}$ TeamSpeak is a voice-over-Internet Protocol (VoIP) application for audio communication between users on a chat channel. ${ }^{7}$ Community Ambassadors are high school volunteers who serve as assistant moderators on the server.
} 
tivities designed to maximize conflict and interdependence. We hypothesized that ownership over the creation, negotiation, and enforcement of community norms would increase youth's sense of agency and belonging, help manage behavior, and contribute to the development of social competence. Specifically, we were interested in identifying and reporting actions taken by youth and moderators toward managing behavior in their community. What kinds of approaches did they use to manage their behavior and resolve conflict, for example? What relationships, if any, formed out of these actions?

4.3.1 Community-based Activity Structures As Surrogates For Technological Controls. Minecraft servers typically rely on a combination of legal instruments and technical solutions to manage player behavior; players are rarely given room to organize themselves [152]. In analyzing the data for Study II, we looked to identify and report actions taken by youth and moderators to manage player behavior within an environment designed explicitly to support player organizing without reliance on more typical forms of technical control. Our findings suggest two overlapping patterns of action and interaction by moderators and players. The first pattern shows moderators shifting from a reliance on discussion-based interaction with youth to an approach that relied on the design of activity structures enabling youth to resolve differences or tensions themselves. For example, in Week 5 when one of the teams was divided about a decision of whether or not to allow another player to join their team, Mod2 suggested that players could vote and decide based on majority, but took no action beyond that to structurally facilitate team formation. By Week 14 moderators had introduced an explicit "crew system" where youth were tasked with forming groups amongst themselves for completing the planned quests. Similarly, over time moderators took on a more peripheral role whenever tensions or conflicts occurred during the gameplay. Rather than intervening during play, they used opening and closing rituals as the space to facilitate discussions among youth by asking open-ended questions (e.g., Mod 1: "If someone didn't cooperate with you, and refused to work with you, how would you deal with that?'). This shift away from ad hoc intervention-style interactions toward a more consistent reliance on structural features to help manage player behavior was an emergent feature of the program's design. Community-based activity structures emerged as effective tools to control player behavior and manage conflict, serving as surrogates for more typical technological controls. As in Study I, our findings suggest the benefits of reframing moderation approaches toward structures in which youth participated actively in problem solving.

The second pattern showed an increase in player actions and interactions associated with making friends and reinforcing community bonds as youth were given increased ownership over community management. We note that select youth settled into leadership roles (e.g., P1, P7, P12, P14, and P20) during the first half of the program when everyone was assigned into moderator-created teams. Within teams interpersonal dynamics appeared formal and transactional, reliant on a handful of typically more experienced players to guide team activity. In contrast, once players were given the opportunity to form their own teams during the second half of the program based on who they played and/or solved quests with, interpersonal dynamics and player actions appeared to support social bonding, and in some cases friendship. For example, P6 offered items such as crafted swords or food to P2, P4, and P8 as giveaways, and P2 often sought to play together with P8, P15, P16, and P17. Similarly, a possible bond was formed between P9 and P12, as P9 looked after their items while P12 was away for a few weeks, instructing new players not to disturb P12's setup. P2 and P9 took actions to integrate new players into the social community, volunteering to help welcome new players during weeks $15-20$ by giving them a guided tour of the virtual world and offering to play with them. Our findings suggest that players, when given the opportunity to 
manage their own relationships and make decisions about their community they did so in ways that built social capital and strengthened social bonds.

We observed that both of the patterns described above were slow to develop, hindered in part, by moderator attitudes. While the moderators in Study II operated within a context that tried to explicitly communicate and reinforce through its design that youth were capable of contributing to server governance, data shows that it took time for moderators to trust in the abilities of youth to manage their behavior and self-organize, without the help of technological safeguards. For example, the traces data show that it took until week 14 for moderators to support the desire among youth to create their own teams. Similarly, it was primarily in the last 6 weeks of the program that we observed moderators deferring to players to discuss and address any tensions that surfaced. Concurrent with these changes, we noted that players increased their use of text and voice-chat to communicate and talk over differences either 1-1 or during small group discussions. This is evident in our audio traces captured both by the researcher present as well as by automated "bots" that followed players who moved across the TeamSpeak channels more freely in the second half of the program to talk with other players. We also note that although moderators eventually deferred the decision-making to the players, they were always present and available-as evidenced in the moderator chat logs. They would respond to players that asked for help but also used opening and closing rituals to encourage players to reflect on what they might do if moderators were not around.

4.3.2 Evolving a Play Community. A profile of participant engagement shows that CAs were created, revised, or deleted by individuals, small groups, and through larger group consensus Figure 4(a). For the most part, consistent with the approach to moderation outlined in Study I, youth were empowered to continually reflect on the efficacy of the agreements in guiding and managing player behavior, adjusting them as needed. Only when participants seemed to struggle with enacting the norms or resolving interpersonal differences, did the moderators intervene. Analysis of the 35 CAs generated by youth over the course of the program show the agreements played a critical role in helping the youth develop the foundations of an online play community uniquely suited for the goals and aims of its participants. Youth created four different kinds of agreements: agreements related to (1) general social etiquette, (2) Minecraft etiquette, (3) Minecraft play, and finally, (4) norms for playing together (See Table 5). Figure 4(b) shows the overall distribution of codes applied to the 35 CAs.

Agreements guiding general social etiquette offered guidance for player behavior in a group setting. These CAs, like "Be kind" and "No bad words," mirrored the kinds of norms young people would encounter in off-line group settings, like classrooms or after-school programs. Agreements that fell into the category of Minecraft etiquette were game-specific, and described player behavior within the game of Minecraft. CAs related to Minecraft play functioned as "house rules"-unofficial modifications to official game rules adopted by individual groups of players-including rules like, "Ask permission before building on someone's land." Playing together CAs described how one should play with others, in Minecraft or elsewhere (e.g., "Communicate and cooperate with teammates" and "Be mindful of the environment so we don't run out of resources").

An analysis of the distribution of CAs across these four categories over time shows a parallel growth in CAs focused on Minecraft etiquette and norms for playing together (Figure 4(c)). We might theorize that norms related to general social etiquette and Minecraft etiquette form the foundation for growing an online server community. However, for the server community to develop further, social norms supporting productive group play must be encouraged. When the Survival world "reset" with the introduction of a new biome in week 14, the data shows that youth revised their CAs in ways that reflected their evolution as a play community. In weeks 14 and 15, we 


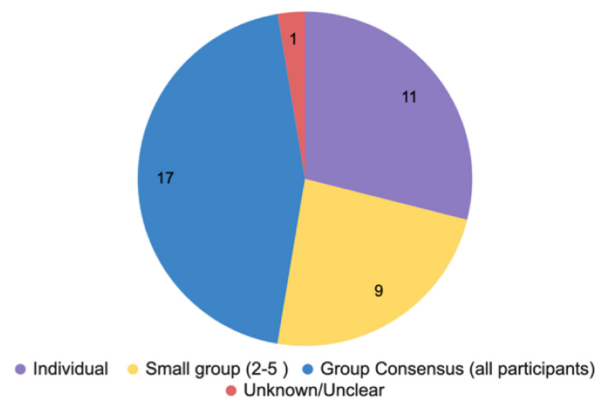

(a) Origin of CAs (individuals, group discussion ...)

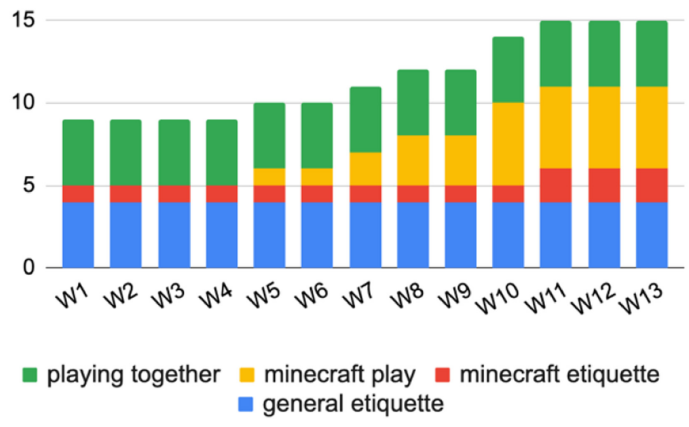

(c) Change in the distributions of CAs by category over the 20-week program

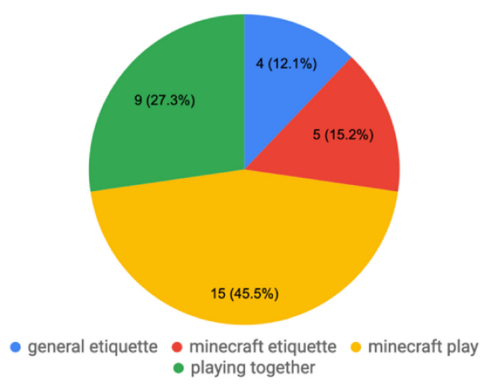

(b) Distribution across four CA categories

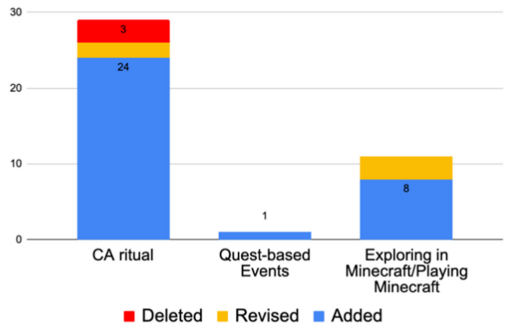

(d) Triggers for the addition, revision, or deletion of CAs

Fig. 4. Source, distribution, and changes over time of the CAs generated by youth during the program across coded categories.

Table 5. Types of Community Agreements

\begin{tabular}{ll}
\hline Code & \multicolumn{1}{c}{ Definition } \\
\hline General etiquette & $\begin{array}{l}\text { How one should think and act in general as a group in the online } \\
\text { space (e.g., be kind, no bad words..."proper and polite behavior as a } \\
\text { member of a group"), not specific to Minecraft }\end{array}$ \\
$\begin{array}{l}\text { Minecraft } \\
\text { etiquette }\end{array}$ & $\begin{array}{l}\text { How one should generally think and act within the game (e.g., ask } \\
\text { permission before building on someone's land; "proper and polite } \\
\text { behavior as player on a shared Minecraft server"), specific to Minecraft }\end{array}$ \\
Minecraft play & $\begin{array}{l}\text { How one should play Minecraft (e.g., no PvP without permission, not } \\
\text { pushing someone in the void,); rules for play that are specific to the } \\
\text { game }\end{array}$ \\
Playing together & $\begin{array}{l}\text { How one should play with others (e.g., be nice to others, communicate } \\
\text { and cooperate with teammates) applicable in general when playing } \\
\text { with others and not just Minecraft }\end{array}$ \\
\hline
\end{tabular}


see a significant increase in CAs related to playing together, paired with a significant reduction in general etiquette CAs. While CAs in the general etiquette category served youth well in the early weeks of the program, they were replaced by norms tied to the needs of players cultivating a specific Minecraft community.

The trends we observed in the types of CAs created and held by youth in the program, while specific to Survival Lab, suggest that use of CA rituals provided a necessary scaffolding for youth to develop governance for their community. Data show that most CAs were added, revised, or deleted as part of the CA rituals embedded in the program (Figure 4(d)). As we consider ways of scaling the program, there is a need to more deeply understand the specific role played by these mechanisms relative to features like the size of the overall community, the length of the program, and the needs and desires of the youth that make-up the server community.

Finally, the traces data revealed that the CAs were not the only source shaping player behavior within the play community. In some instances participants modeled values that were not explicitly stated in the agreements. These included, for example, offering gifts when someone helped them (e.g., P11 and P14); being generous by paying for items that a peer was giving away for free (e.g., P6, P7, and P15); and giving peers the benefit of the doubt amidst intrapersonal tensions (e.g., P1, $\mathrm{P} 4, \mathrm{P} 6$, and P11).

Over time, Survival Lab became a place where they felt safe and purposeful, and where their play felt important, relevant, and valuable, as reflected in their CAs [83]. The traces data show that participants generated most of the agreements fairly quickly during the CAs rituals, without help from the mods. We observed participants sharing their ideas on Minecraft chat and/or Teamspeak voice as soon as the moderators prompted them to discuss the CAs. While half the agreements reflect participants' expectations for game play (e.g., that others should not grief their land), the other agreements show that, when given the opportunity, youth were able to reflectively formulate more strategic agreements aimed at long-term visions for the community (e.g., being mindful of resources and cooperating with each other). The process of establishing, reflecting on, and revising agreements gave youth a simple way to express their ongoing commitment to membership in the community.

4.3.3 Community Agreements as Tools "to think, act, and play with". Across 5,000 hours of log data we observed surprisingly few conflicts (See Appendix 7), at least of the kind profiled in Study I, which would require some kind of "formal mediation." While we cannot claim that the use of CAs led to a reduction in conflict of this type, we did observe youth engaging purposefully with CA as tools to think, act, and play with. Youth used CAs as a tool to increase their chances of successfully completing in-game quests; to foster social relationships and strengthen community bonds, and to maintain a positive community climate by limiting toxic interactions. Participants created CAs that served both as proxies for game rules (e.g., No TNT cannons; No PvP without permission), as guides for social behavior (e.g., Don't boss other people around), and as expressions of community values (e.g., Always be honest with others). Data show that in some cases, youth developed CAs that reflected not only a shared sense of purpose but also a commitment to supporting a diversity of interests and goals within the play community. The introduction of an oasis of virtual cows in week 7 provides one such example.

The traces data show that immediately upon the unveiling of the oasis, several players began killing cows in an effort to earn experience points (XP), which would help them in the future. Two players were upset by the killing and one of these players (P9) then engaged in player-versusplayer or PvP combat to protect the cows. One of the moderators (CA1) suggested that the players talk it out rather than fight, repeatedly reminding them of the CA against PvP. When the player 
continued to fight, the moderator activated a command that immobilized the player's character for a short duration, expressing their reluctance to do so the other moderators (CA1: "I hate to freeze people on this server, but it feels like P9 is starting conflict just to get the attention of a 1-1 talk').

During the opening discussion the next day, moderators asked the community if they wanted to discuss their differences of opinion and possibly form a new CA that could govern their interaction with the cows. The youth seemed divided in their views on how to utilize the cows, which are valuable resources in the Minecraft world. Eventually, the group agreed on a rule that the oasis cows could be killed during the day but would be protected at night. This decision cleverly incorporated both the short-term and long-term goals of most participants (e.g., earn XP; save resources, respectively), while also benefiting the community overall increasing their chances of successfully completing in-game quests. Even after they agreed on the new rule, we saw that players remained sensitive to each other's concerns and needs (e.g., P14 reassured P7 that they could earn XP in other ways), which served to reinforce community bonds.

In addition, youth created a new CA: "Be mindful of the environment so we don't run out of resources." While we cannot claim a correlation between the group's decision to establish an agreement limiting how a particular resource could be claimed (killing cows), we can speculate that CAs created a context for participants to reflect on a larger set of values they held for their community. In creating an agreement that encouraged players to attend to the present environment as a way to ensure the possibility of ongoing game play, we can see participants going beyond their own self-centered needs, extending outward to the pursuit of goals that benefit the (virtual) world.

4.3.4 Giving Youth Voice is more Effective when Youth can see that Someone is Listening. While findings from Study II focus primarily on youth-led governance, strategies for empowering youth are not limited to norms or agreements alone. Supporting youth in voicing their ideas or opinions, defining their own goals, and deciding what activities to undertake can be equally effective. Youth in Study II were given opportunities to decide for themselves how to participate in the team-based challenges and group discussions. For example, a handful of participants (e.g., P7, P8) declared that they were "bored" on a few occasions and either left the session early or decided to go adventuring in the virtual world on their own. The youth were not chastised either by the moderators or by other participants for choosing an alternative activity or logging off.

Moderators were attentive to times when youth appeared to be tired or distracted, responding by planning spontaneous activities or asking participants to suggest ideas for an upcoming event in the game. For example, in Week 12, having heard the participants' wish to engage in playful $\mathrm{PvP}$ battles, the moderators arranged a temporary "PvP arena" for interested players. The same day, the moderators also asked participants for suggestions on how to improve Survival Lab and used the ideas to refine the program's design. When the new Space Biome launched in week 14, participants acknowledged and appreciated that it reflected some of their input (e.g., making food harder to obtain, fewer creepers, and mob griefing). The traces data showed that the moderators also introduced a new "crew system" for forming teams in the Space biome, an idea based on P12's suggestion (P12: “I think a 'family system,' too"). Similarly, in week 13, the moderators replaced the usual team-based challenge with a game "Blocktionary" (like pictionary with 3D blocks in Minecraft) to address signs of boredom that participants showed earlier in the session. The spontaneous and flexible approaches adopted by moderators reinforced the idea that youth voice mattered in Survival Lab.

4.3.5 Summary of Findings. The findings from Study II are promising and encourage a closer look at the ways specific design affordances impacted player behavior and interactions on the 
server. Moderator-facilitated CAs became a mechanism by which the community managed player behavior on the server, for example, becoming surrogates for technical controls that more typically control behavior. Rather than relying on moderators to "keep the peace," youth used CAs (and the process of their design) to increasingly tailor their play to reflect their interests and values, strengthen social bonds, and sustain the community. CAs both informed, and were informed by the experiences of youth on the server. Specifically, the data suggest that giving youth ownership over the creation and maintenance of agreements in a context where adults listened to their ideas was a crucial feature of the play environment; as was providing consistent opportunities for youth to reflect on and revise CAs in response to changes in community membership or specific in-game experiences. Positive adult-youth relationships formed as a result. Moderators played a key role in prompting ongoing reflection through well-designed opening and closing rituals and in-promtu discussions held in response to conflict events or behaviors. Overall, these design decisions seemed to empower youth to determine and pursue both individual and collective goals, which helped to foster ownership of community moderation and a real sense of belonging. As youth engaged with others around a shared interest, they were able to see the importance of their contributions to the community, which added meaning and further motivated positive participation [156]. We now turn to examine how these findings relate to the broader set of goals guiding this work.

\subsection{Study II Discussion}

The aim of Study II was to expand on the initial findings of Study I, which suggested the possibilities of less authoritarian mediation techniques to increase the agency and problem solving capacities of youth in resolving communal and interpersonal problems. Study II thus moved from the use of a purely moderator-facilitated support into embedding these approaches into the design of the learning environment itself. The design of the program-which took place on a custom Minecraft server-would minimize moderator involvement, remove any traditional technology solutions (designed to prevent conflict), and maximize youth engagement in defining and maintaining the social norms of the community. To do so we drew on two well established offline community-based practices: the use of CAs as a youth-led governance structure and recurrent "rituals" that structured and facilitated communal reflection; both underpinned by the problemsolving moderation (ask rather than tell) approach. The findings described above-centered on the interactions with CAs as the key design mechanism-revealed the multitude of ways in which youth used CAs and the discussions around them to gain control over the environment, diffuse and dissuade toxic interactions, reinforce social bonds, and support the interests of participants. The findings suggest that moderators played a facilitative, rather than authoritarian role in encouraging youth to operationalize (and grapple, if necessary, with) the CAs, and allowed the youth take responsibility for the social climate of their online play community. Our findings are consistent with prior work on "student voice," (e.g., [53, 124, 125]) which underscores the critical role adults can play in empowering students. While there may have been a few instances when moderators intervened to settle a dispute (e.g, between P9 and P2; P9 and P8) or suggest a new agreement be added (e.g., oasis cows), they did so in ways that entrusted the decision-making to the youth participants. The moderators also invited and incorporated suggestions from youth into the design of the virtual world and the game play events. Our findings begin to suggest that youth can be effective at self-governing when given the tools to do so, and this experience in itself can be meaningful.

The study of socio-technical systems to better understand how we might design for increasing social capital (e.g., [9, 29, 145, 163]) has remained a topic of great interest in HCI. Prior studies 
$[148,158,159,172]$ have established our understanding of technological features used to help moderators govern online communities (e.g., automatic bans, virtual jails, and freezing players in the game). Whereas Survival Lab design included technology-mediated audio communication through tools like TeamSpeak and rituals such as reflective discussions, CAs, and game-play quests aimed at increasing social interactions among the youth. Our analysis of the traces data shows that this setup made "social practices" [145] more possible as youth collectively participated in generating CAs, discussed and improved interpersonal rapport and teamwork, and self-organized into groups to complete the assigned quests. Our findings support prior conceptualizations in $\mathrm{HCI}$ that the design of socio-technological systems is often asymmetric. By eliminating standard technological controls and enriching the social infrastructure in the form of caring moderators, technology-mediated communication tools, and contextual narratives (i.e., gameplay affordances of Minecraft to support time-sensitive quests), we found evidence for newer possible models of online governance in play-based communities like Minecraft.

The approach taken in this work is analogous with that undertaken by community-based prevention science programs like the 4Rs Program, "Reading, Writing, Respect and Resolution" [90], a school-based SEL and literacy development intervention. As in our work, 4Rs focuses on the use of "small" tools and interaction techniques that encourage youth to reflect on conflict, associated feelings, and decision-making. By drawing on this scholarship-and examining how such tools and techniques could be embedded into online learning spaces-this work aims to explore ways of explicitly designing for youth agency and interest, while also maintaining the safety and security of participants. It has sought to challenge existing online approaches to the design and moderation practices of many "kid-friendly" online communities, which limit and control youth participation and social interaction, and which we see as limiting the capacity of youth to develop and grow their social competence. Following, we reflect on the two studies presented in this article and discuss the broader design implications of our work.

\section{GENERAL DISCUSSION}

The work presented in this article presents the findings of two online after-school programs in Minecraft: KC and Survival Lab. Both the ICPS-based approach to moderation in KC (Study I) and the use of CAs in Survival Lab (Study II) explored ways of empowering youth to take responsibility for (and resolution of) the interpersonal interactions they experience online. The ICPS dialoguing approach used by moderators was intended to increase youth agency by helping them to identify potential solutions to problems that arose on the server, including interpersonal conflict, while also serving as a mechanism by which a problem solving approach to conflict was internalized by the community itself. While moderators facilitated the dialoguing process, asking questions which encouraged youth to reflect on their own behaviors and how those behaviors might relate to others' feelings and experiences, it was ultimately incumbent on youth to identify ways to resolve the conflict. In this sense, Study I was our first foray into understanding how online interventions could be designed explicitly (e.g., through moderator training) to enable the development of social competence, or deliberately target youth empowerment and agency.

Study II, on the other hand, examined the effects of an easing of technical controls in favor of social ones, an emphasis on cooperative and interdependent play, and the use of CAs, in the context of an ICPS-like approach to community moderation. Our findings suggest that over time, youth conceptualized Survival Lab as an egalitarian online community, as they partook in collective decision-making to define, enact, and revise formal agreements guiding their play together. Revisions to CAs suggest that youth felt a sense of belonging to the community and observations of game play show youth acting with autonomy. We note that in Study II, while moderators 
did not always remember to fully use the ICPS approach piloted in Study I, they demonstrated a problem-solving mindset: giving youth opportunities to reflect on productive responses to conflict, for example, and refraining from "swooping in" when conflict arose. As in Study I, the moderation approach used in Study II played a key role in the outcomes observed: the ICPS-like moderation helped enable the development of an egalitarian community, gave moderators the ability to provide suggestions (but not to overpower youth), and provided youth participants with tools to scaffold their reflection.

\subsection{Design Implications}

While the results of these small studies are promising, they also highlight some of the challenges of creating and sustaining online environments that are truly youth-centered. Both the ICPS dialoguing approach to server moderation, and the use of CAs and other reflective practices demands a significant shift in mindset, for both adults and youth. Adults must overcome a tendency to control, direct, or correct youth, while youth must see themselves as capable of solving problems and resolving conflict, rather than relying on adults to intervene. Online play-based settings like those profiled here can provide a safe space for practicing such shifts in mind-set, while also offering youth authentic experiences in which to develop problem-solving skills and increased social competence. Future interventions targeted in similar settings will need to consider ways of supporting adults in making this shift in mindset, while offering youth opportunities to exercise their agency and make decisions on their own.

This does not imply that no adult intervention is ever needed; some conflicts or problems necessitate adult intervention. Rather, we mean creating contexts and opportunities that help youth (1) embrace conflict, rather than avoid it; (2) recognize that they have values that contribute to the growth of communities they participate in; and (3) help youth recognize their ability to problemsolve with others. Taken together, findings from Study I and II show that moderation alone may be insufficient to drive desired outcomes of PYD. Only when combined with mechanisms that promote youth voice and encourage self-determination, such as CAs and reflective discussions, does moderation play a critical role in reinforcing these outcomes.

These challenges emphasize the need for a closer look at social, user-driven models of community moderation and management. We have identified several potential future areas of work. They include:

-Developing new kinds of moderator tools: Platforms should consider features that encourage positive behaviors in meaningful ways. While tools for dealing with misbehavior are common, tools for encouraging meaningfulness are limited at best (e.g., Reddit gold and Facebook reactions). How are platform developers balancing punitive (i.e., auto-banning bad behavior) and preventive approaches (i.e., modeling behavior and rewarding those who help make the community better)?

- Exploring prosocial interaction and incentive systems: How might interaction and incentive systems be designed as levers to either curb negative player interaction and communication or nudge prosocial behavior? These include things like the design of combat systems, matchmaking systems that group players, and mechanics and incentive systems encouraging collaboration, shared planning, or teamwork. Research could focus on how player behavior might be shaped by the interaction and incentive systems underlying their play. Studies in offline settings $[121,134,135,182]$ reveal what design strategies around social norms and the role of community role models are helpful in encouraging prosocial behaviors. Our work offers preliminary but foundational evidence that offline design strategies 
can be translated into online settings. Future work could examine and evaluate such interventions.

- Scaffolding opportunities for player-driven community governance: Online play communities for youth should consider how to scaffold opportunities for players to engage in discussion, reflection, and decision-making about the rules and norms of their community. How might social features be designed to provide ongoing opportunities for youth to take leadership roles in resolving conflict, for example?

\section{CONCLUSIONS AND AN EMERGING RESEARCH AGENDA}

Current mediation practices aimed at minimizing or eliminating toxicity in online spaces tend to normalize punitive measures or adopt deterrence approaches as ways to deal with conflicts and interpersonal problems that youth may encounter in online social settings. Such approaches, while effective in limiting toxicity, are mostly ineffective as tools for youth development. Our research has shown evidence-based offline practices originating in Prevention Science and the learning sciences can be adapted for use in online play-based settings. While moderators in our work were trained and committed to supporting youth empowerment, more work is necessary to understand any discrepancies between online mediation strategies that adults (e.g., parents) perceive to be effective and those that effectively support youth development.

To the best of our knowledge, this work is the first to examine whether the effects of offline strategies like ICPS dialoguing or CAs transfer into online game-play. The results suggest that such evidence-based interventions can be adapted to online settings. While substantial future work is required, these early results open up an intriguing novel research agenda exploring the possibility to deliver social-emotional interventions within online settings. Future work would require ongoing collaboration among research scientists and developers in game design, artificial intelligence, machine learning, data and learning analytics, and social network analysis with experts in child development, community psychology, interaction design and children, learning, and play. Goals would include developing guiding principles around ethical and effective types of models, algorithms, and analytics to be used, inclusive design processes, ways of addressing barriers, and methods of effectively measuring impact, as well as recommendations as to the types of data to be collected, with privacy concerns in mind. These principles can be used by designers, developers, policymakers, researchers, and funders of youth-friendly online environments supporting the social-emotional development of youth and families.

Advancing this agenda will require work on a number of related issues, three which we briefly discuss here: (1) understanding youth motivation and social connection, (2) creating connections across settings, and (3) enhancing and limiting factors of scale.

\subsection{Understanding Youth Motivation and Social Connection}

Children learn best through learning that is interactive and relational, and which takes place within an emotionally supportive environment [131]. In such environments, “...youth feel safe to try, fail, and work together to overcome obstacles and learn from their mistakes" [111, 128, 131]. Online environments can be designed to offer learning experiences that are interactive and relational, as shown in our findings, but much more work needs to be done to understand youth's motivations for engaging in such spaces. Research on online affinity networks-which are organized around a shared interest-provide one model for understanding motivation and engagement [85], but more models are needed in order to be able to better tailor other offline strategies to online settings. 
While the IRB constraints inherent to the case study server prohibited us from interviewing youth, future work can take on other methodological approaches.

In addition, future work should engage with data analytics and machine learning to develop a richer understanding of the kinds of social connections and conflicts youth develop in online playbased environments focused on promoting SEL and development. Research questions guiding this work might include: How might data analytics be used for studying youth interactions in online communities? How might machine learning be used to better understand the kinds and qualities of social interactions children are having when they play together online? What role might data analytics play in informing the design of online environments promoting SEL and development?

\subsection{Creating Connections Across Settings (Transfer)}

We argue that informal play-based online settings lend themselves as rich sites for youth to engage in interest-based activities with peers and other adults. Questions of how skills developed in one setting (online games) might transfer to others (home or school settings) will need to be a part of a larger research agenda guiding future work. What is the extent to which online environments are effective in facilitating lasting development of social competence and problem-solving skills? Do these skills transfer to out-of-game contexts? Several key variables are at play: we do not yet understand, for example, how youth conceptualize their participation in programs such as $\mathrm{KC}$ or Survival Lab relative to their participation in other online and/or offline settings. This understanding can offer a more holistic perspective on how problem-solving and social skills can be developed across settings. More work is needed to identify and describe the most favorable conditions and appropriate structures of communities for successful translation of offline evidence-based strategies into online settings. We acknowledge the obvious fact that play-based online settings are not intrinsically motivating for all youth and/or adults. This leaves open questions around how nonplay based online settings, particularly those involving real-time social interactions, might provide similar opportunities to translate offline evidence-based interventions.

\subsection{Enhancing and Limiting Factors of Scale}

Online play-based communities often involve massively large online communities. While our interventions were focused on smaller communities, future work will need to understand the challenges in translating evidence-based offline strategies in such large communities. In terms of scaling the alternative approaches presented in this article to larger communities, we encourage caution as we do not fully understand what are the enhancing and/or limiting factors that might exist. To quote Bruckman's position [25], in the context of community size, "...Larger communities necessarily become bureaucracies; in a real sense, they cease to be communities at all." (p. 2). However, both PYD and SEL offline interventions have been scaled at least at the level of large schools, with limited resources, which is encouraging.

\section{APPENDICES}

\section{A STUDY I: KID CLUB}

\section{A.1 Server Information}

The non-profit organization running the server, provided the authors access to the full logs of communications during its 18 months long history, dating to summer 2015. Within this article, we sampled a subset of the KC logs for analysis, originating from two main periods: first we sampled a random subset of 30-day logs (each 8 hours long) from the 2 months long period between June and September 2015, yielding 225 conflicts-referred to as Summer'15. Second, we included all logs 
between December 28th, 2016 and February 28th in 2017 (i.e., 62 logs, each 2 hour long), yielding 76 conflicts-referred to as the Winter' 17.

For each of these day-logs, a trained Research Assistant read through the transcript, identifying instances of conflicts through using a series of keywords that were developed by the team, as already reported in Slovak et al. [170]. The identified conflicts were then marked in a cloud-based software Dedoose ${ }^{8}$ and underwent further qualitative coding. This was crucial for developing a qualitative understanding of the types of conflicts and the moderation strategies on the server. In particular, each conflict excerpt was labeled to indicate the predominant type of moderation used throughout the conflict according to the Prevention Science typology (power, suggestion, explanation, problem solving; [164, 169]) as well as additional qualitative indicators of the type (youthvs-youth, youth-vs-community) or nature of the conflict (physical, emotional, social). These data were further supported by interviews with moderators-see [170] for more details.

\section{A.2 Dataset Used in this Work}

To answer the research questions posed in this study, it was necessary to code the available excerpts with much more granularity: instead of operating qualitatively on "full" conflict instances, we required coding the excerpts on the level of individual utterances to examine how the type of moderator's statements impacted youth's responses. The coding procedure is described below.

A.2.1 Sampled Conflicts. We coded all of the 76 conflicts from the Winter' 17 period. In addition, we also randomly sampled a set of additional 30 conflicts from the 225 conflicts identified in the Summer 2015 period. The aim was to explore whether any observed differences are stable across time and changes in the moderators/youth on the server. As the Summer'15 period was predominantly skewed toward power approaches (69\% of all conflicts) with no problem solving, a random selection without stratification would likely oversample power utterances. As such, we stratified the randomization based on the conflict-level categorization described above (cf. [170]): we randomly selected 10 conflicts labelled as "predominantly power"; 10 labelled as "predominantly suggestion"; and 10 labelled as "predominantly explanation." Each conflict logs across both of these periods was first 'cleaned' to include only utterances of those involved in the specific conflict (as many other conversations could have been interleaved as is common on busy servers). We then coded the moderators and youth statements separately.

A.2.2 Coding Scheme for Moderators. The utterances by moderators were coded with either one of the four conflict resolution methods categories drawn from Prevention Science theory [164, 166-169, 175]-power, suggestion, explanation, problem solving - or a "non-method utterance" category that included clarification requests (e.g., "what has been griefed?") and appreciative statements after the conflict was resolved (e.g., "thank you" or "it's okay"). Specifically, the resulting coding scheme was theoretically grounded in the I Can Problem Solve framework to define the four categories and drew further on our previous qualitative work to operationalize these theoretical concepts into the Minecraft context (cf. [170]). The summary of the resulting coding scheme is outlined in Figure 5. Every utterance was coded by two researchers; any disagreements (less than $1 \%$ of utterances) were resolved by discussion.

A.2.3 Coding Scheme for Youth. Development of the youth statements' coding scheme was less straightforward as, to the best of our knowledge, there is no existing categorization that could be directly applied. The Prevention Science theories provide some assumptions and expectations as to what could be considered positive responses (e.g., increased agency in problem resolution and

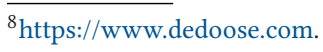



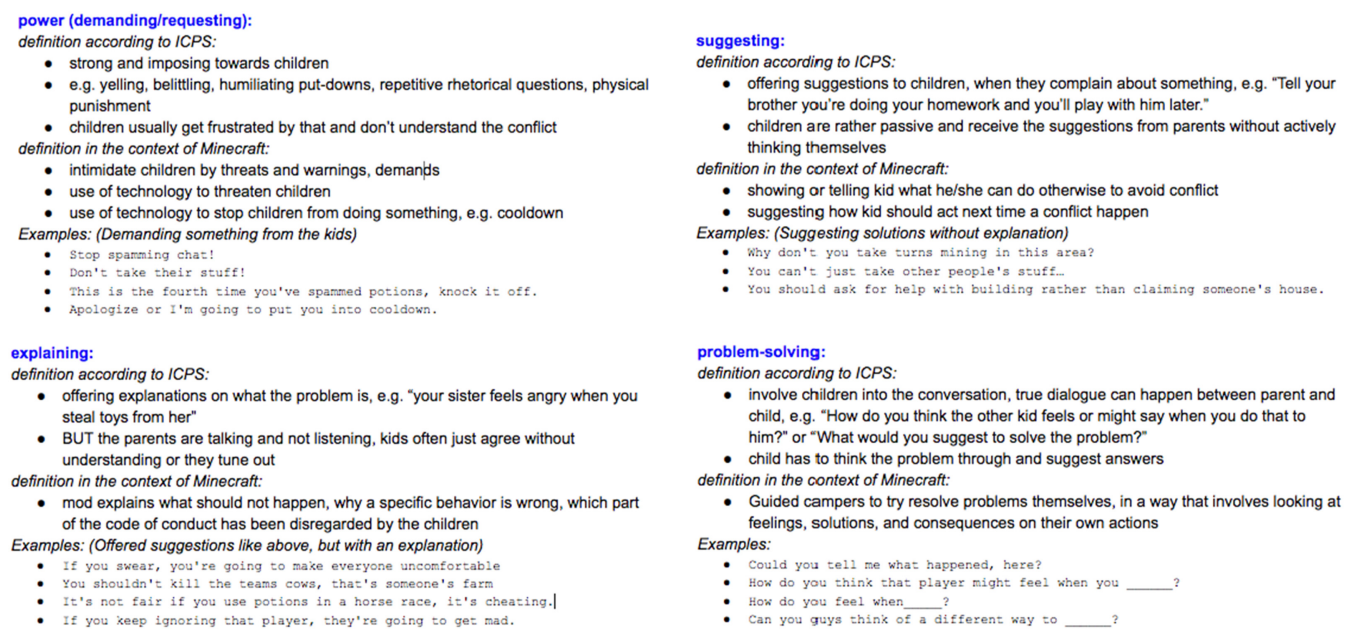

Fig. 5. Coding scheme for moderators.

reflective thinking), as well as what the negative responses might be (e.g., shouting, ignoring the problem, crude language). However, it was not clear what a full classification of responses could be, especially as no prior literature has analyzed the conflict responses within the online context. Our approach therefore relied on thematic analysis as an inductive method outlined in [24]: The last author and a research assistant (Stephanie Ta, in acknowledgements) have independently read and re-read the youth utterances to familiarize themselves with the data. Following this, initial codes were generated across the dataset and sorted into potential codes, still independently, and all the relevant coded data extracts within the identified themes were collated. At that stage, the first and last author worked together to review and refine the initial themes both at the level of the coded extracts, as well as in relation to the entire dataset. Codes were then refined, generating an initial thematic map of the analysis with the resulting hierarchy of codes and sub-codes. This process was followed to as certain that the generated hierarchy and individual codes accurately represented the data, and that the collated extracts for each code formed a coherent and consistent pattern across the dataset. The refinement of the coding hierarchy and individual codes involved several iterations, until consensus was reached among both authors that each utterance is correctly coded. The result was a hierarchical coding scheme, with nine first-order themes: reflection; making up/aftermath; clarification request/fillers; factual disagreements; explaining behavior/intent; short answer; deflecting; pleading; and uncooperative behavior.

Finally, the nine first-order themes were combined into three categories (positive, negative, and neutral) corresponding to the perceived value of the youth response in response to the prevention science framework. Our aim was to categorize a label as "positive" or "negative" only if every interaction under that label would clearly be constructive/non-constructive; accepting a larger neutral category as a result. Specifically, only the responses that represented explicit steps toward resolving the problem by the youth were labelled as positive (i.e., reflection, making up/aftermath); similarly, only the responses that represented a clear breakdown in the interaction were classified as negative (uncooperative behavior, deflecting, and pleading). The remaining set of responses was classed as neutral, as these could be seen as negative or positive depending on context: for example, "explaining intent" could be seen as constructive conflict resolution step if the youth is explaining their actions to illustrate how a misunderstanding occurred; or negative if they are repeating the 
same "explanation" multiple times without actually engaging in the discussion. The full coding scheme for utterances by youth is provided in Section A.2.5 below.

The finalized coding scheme was further verified by a separate researcher: the second author was trained in using the codebook by first coding a subset of the data under supervision ( $30 \%$ of utterances); and then coded 50\% of the dataset fully independently. The Cohen's kappa scores and $\%$ agreement on this independent coding (computing using a standard package (IRR) in R) showed a high reliability with kappa $=0.965 ; \%$ agree $=97.1$.

A.2.4 Computing Impacts of Moderator Utterance. For the final measure of moderator impact, we gave each moderator utterance an overall rating based on the content of the subsequent youth responses up until the moderator's next utterance. If the youth made at least one negative response, we rated the moderator utterance as having a 'negative' outcome; if the youth made no negative responses but at least one positive response, we rated the moderator utterance as having a 'positive' outcome; otherwise we rated the moderator utterance as 'neutral'".

A.2.5 Line-by-Line Coding Scheme for Child Utterances. We outline the resulting scheme, already clustered into the three overarching codes: positive, negative, and neutral.

Positive: Top level code for instances where the kid demonstrates thoughtfulness and in general a positive attitude. The kid is responding to/thinking about (reflecting) the conflict/problems, solutions, feelings (their own and that of their peers) in ways that help move the situation forward. This code also covers instances where the kid is trying to make up for an action/something they said to a mod/peer or acknowledging another kid's apology.

- reflection: The child utterance shows active engagement with situation/conflict on an emotional or interpersonal level. This can involve insights into the situation itself and its consequences, the participating people and their perspective/emotions, own behaviour and emotions, and possible future implications. Typical examples include:

- plan for future, e.g., "alright, I'm not gonna" (as in the kid understood the problem and gives notice on how to act properly in future conflict situations)

- reflection: consequences from others, e.g., "because, then other people will get mad at you"

- reflection: current conversation, e.g., "you asked me a question and I gave you an honest answer"

- reflection: emotional state, e.g., "I'm sad, everyone is picking on me", "i feel like donesnt trust me," "I was mad and triggered because some girl on the internet is being a turd"

- reflection: own behaviour, e.g., "I know I should stop," "What I said was not a good response"

- reflection: perception of others, e.g., "they did not tell me," "they are just mean"

- reflection: suggested resolution, e.g., "we can give it back and say sorry" (as in the kid understood the problem and gives notice on how to act properly in the current conflict situations)

- making up/aftermath: The kid is insightful; reconciliates with and/or apologizes to either mod or other kids. This is so to say the first step to problem-solving but is not yet a state of reflection, but rather the awareness of being. Some examples include:

- apology to mod

\footnotetext{
${ }^{9}$ We went with this classification due to the common notion in social and cognitive psychology that negative responses tend to be more cognitively salient than positive ones [15]. In only three cases, a moderator utterance was in fact followed by both negative and positive responses.
} 
- apology to agrieved camper (agrieved camper) content with kid, e.g., "you're fine”

- "im sorry im just still a little mad"

Negative: Top level code for statements where the kid is rude/insensitive/non-cooperative and saying things that could worsen and not resolve the problem or if the kid is already banned/in a cooldown but wants to get out (pleads with the mod). Usually, these utterances occur just when/after a conflict/tension has surfaced, or if a mod is persisting to ask questions/explain things but the kid wants to move on.

- deflecting: Trying to avoid the "harder" discussion by changing the topic or bringing in sarcasm/jokes. Typical examples include:

- "Hmmm idk, maybe give them ANOTHER stack of diamonds? ;-;"

- "Lol Im not really gonna lol"

- pleading: The kid is pleading for help/support from the mod to get out of a desperate emotional state. Kid can both be insightful or not in this situation. But mostly mod is responsible for desperation. Typical examples include:

- "please talk to me"

- "can you unmute me"

- uncooperative: The kid is not trying to cooperate with the mod in the process of problem solving. This is characterized by following behaviour patterns: being inconsiderate, complaining about mod's behaviour, being rude with mod and rejects the mods help or yelling things. Some examples are:

-inconsiderate, e.g., "lol," "I'm going to dump him in lava"

- complaining about mod's behaviour, e.g., "I think the mods should be more fair on me," "the mods won't help"

- cursing/being rude to mod, e.g., "Don't ruin my game experience by being a jerk," "**** you"

- rejects help, e.g., "leave me alone," "I'm done," "never" yelling/exclamations, e.g., "WHAT THE HECK MOD," "HE BROKE ANOTHER ONE”

Neutral: The remainder of the utterances was classed as neutral. As seen below, these were statements that often aimed to seek or deliver clarification for the activity in question: predominantly, the statements are providing descriptive accounts of the situation (even if that means respectfully disagreeing with the moderator). The specific codes were classed as follows:

- clarification request: The kid does not understand his/her fault and is asking the mod to clarify the situation/explain relevant information to them, so that they can follow on what the problem is. Typical examples are:

- "what do you mean?"

- "so should I act out?"

- factual disagreement (to establish common ground): Disagreement aimed at the "problem," trying to establish common ground. Typical examples are:

- (disagreement) “SO WHAT DO I DO WHEN HES BREAKING MY STUFF!!!” (note, this is not a question in the context)

- "Yell at me instead"

- "Sure. Just dont let him kill it" (the kid agrees, but suggests a mod action)

- "... at least put me in the right chat channel" 
- explain behavior: The kid is trying to elaborate their perspective and the intention behind his/her actions. This involves explaining the situation and feelings of how the kid experienced it as well as what he/she see the other kids as doing wrong/right (as well as what he/she is doing right). Some examples are:

- explain logging off (say why they logged/about to log off: "My mom says I have to go to bed. and if you think thats an excuse...")

- complain about others (=explain what others did wrong), e.g., "everytime you turn your back he tries to push me into lava"

- explain situation, e.g., "this is my build," "I had no hunger bar," "I saw him and he suddenly disappeared," "mine does not lag though"

- argue that behaviour was correct (and why), e.g., "I haven't said a single curse word," "I did, he went anyway"

- short answer: The kid is giving a really short answer (letting the mod know that he/she heard what the mod said) without giving information about any new knowledge gained about the situation. The child is passively agreeing/acknowledging something the mod has said, e.g., "no," "yes," "uhm," "k."

\section{B STUDY II: SURVIVAL LAB}

\section{B.1 Lack of Interpersonal Conflicts}

Despite the design of team-based and game-play activities that aimed to amplify the potential for interpersonal conflict (cf Section 4.3.1), the observational and traces data in Study II revealed only very few conflicts-only five conflict instances were observed during the whole 6 months Survival Lab program (40 sessions, twice a week over 20 weeks). Here, "conflict" refers to instances where one or more players had violated a CA or did something else that was at odds with the other players (e.g., killing the oasis cows). Table 6 outlines the conflicts and outcomes for those five instances, including the weeks in which this occurred.

Table 6. Conflicts and Outcomes in Survival Lab

\begin{tabular}{|c|c|c|}
\hline Week & Conflict & Outcome \\
\hline W6 & $\begin{array}{l}\text { Moderators engineered a conflict as } \\
\text { part of the quest (Sandwich-Mayo } \\
\text { episode) }\end{array}$ & $\begin{array}{l}\text { Youth prompted to discuss during the closing } \\
\text { rituals how to organize themselves for } \\
\text { teamwork }\end{array}$ \\
\hline W7 & $\begin{array}{l}\text { P9 strikes } \mathrm{P} 8 \text { in a PvP attack during a } \\
\text { quest }\end{array}$ & $\begin{array}{l}\text { Moderator facilitates a discussion and P9 } \\
\text { agrees to carry out a Community service as } \\
\text { "punishment" }\end{array}$ \\
\hline W7 & $\begin{array}{l}\text { Participants divided on oasis-cows' } \\
\text { killing }\end{array}$ & $\begin{array}{l}\text { Youth create a new CA in the next Opening } \\
\text { ritual }\end{array}$ \\
\hline W12 & $\begin{array}{l}\mathrm{P} 2 \text { and } \mathrm{P} 9 \text { argue based on a } \\
\text { misunderstanding }\end{array}$ & $\begin{array}{l}\text { Participants discuss how to communicate } \\
\text { better during Closing ritual }\end{array}$ \\
\hline W16 & $\begin{array}{l}\text { P8 and P2'S team is broken when P2 } \\
\text { quits to form a new team but soon } \\
\text { changes their mind }\end{array}$ & $\begin{array}{l}\text { Participants agree to wait a few weeks before } \\
\text { they can change teams again and discuss on } \\
\text { better communication during changing teams }\end{array}$ \\
\hline
\end{tabular}




\section{B.2 Community Agreements}

Table 7 lists all the CAs generated by the participating youth between September 2018 and January 2019 in the Survival Lab program.

Table 7. List of all CAs or CAs created by youth in Survival Lab

\begin{tabular}{|c|c|c|}
\hline No. & CAs & Code category \\
\hline 1 & Be Kind & general etiquette \\
\hline 2 & Allow others to speak and listen to their ideas & general etiquette \\
\hline 3 & Please keep a library voice on TeamSpeak & general etiquette \\
\hline 4 & Try to return items to their rightful owner & minecraft etiquette \\
\hline 5 & Be mindful of personal space & general etiquette \\
\hline 6 & Cooperate with others! & playing together \\
\hline 7 & Listen to others if they ask you for help & playing together \\
\hline 8 & Always be honest with others & playing together \\
\hline 9 & Be thoughtful of others' thoughts and ideas & playing together \\
\hline 10 & No PvP allowed. Punishment: Community Service & minecraft play \\
\hline 11 & No cow killing & minecraft play \\
\hline 12 & No hitting randomly & minecraft play \\
\hline 13 & No stealing! & minecraft play \\
\hline 14 & Don't break the train & minecraft play \\
\hline 15 & Please don't spam shop buttons. If you ring one stay at the shop & minecraft etiquette \\
\hline 16 & No griefing, no stealing, breaking blocks, vandalism & minecraft play \\
\hline 17 & Be nice to others and help them when they need it & playing together \\
\hline 18 & No PVP without permission & minecraft play \\
\hline 19 & Return items to the owner if you pick them & minecraft etiquette \\
\hline 20 & Do not use bad words & general etiquette \\
\hline 21 & No TNT cannons. Don't push people into the void & minecraft play \\
\hline 22 & Communicate and cooperate with your teammates & playing together \\
\hline 23 & Don't boss other people around & playing together \\
\hline 24 & Be mindful of the environment so we don't run out of resources & playing together \\
\hline 25 & Ask before you build on someone else's land or area & minecraft etiquette \\
\hline 26 & Do not build traps for other players & minecraft play \\
\hline 27 & Offer to help if someone dies and needs help & minecraft etiquette \\
\hline 28 & You cannot claim entire planets without permission from all crews & minecraft play \\
\hline 29 & (storage Idea 1) Players must earn the privilege to use storage & minecraft play \\
\hline 30 & (storage Idea 1) Players deposit items equivalent to what they take & minecraft play \\
\hline 31 & No deposit is required to withdraw items & minecraft play \\
\hline 32 & Everyone is allowed to use storage if they deposit each time & minecraft play \\
\hline 33 & (safety) Bridges must be built at least 2 blocks wide & minecraft play \\
\hline
\end{tabular}

\section{ACKNOWLEDGMENTS}

The authors would like to thank Mia Doces at Committee for Children for her support of this research, Lynne Maureen Hurdle, the non-profit Connected Camps, as well as the youth participants who made this work possible. The authors would also like to thank Stephanie Ta, Vienna University of Technology, for her help with coding the KidClub data; and Marcel Pufal, University of California Irvine, for his support with the Survival Lab data-collection setup. 


\section{REFERENCES}

[1] [n.d.]. The 4Rs Program | Morningside Center for Teaching Social Responsibility. Retrieved from https://www. morningsidecenter.org/4rs-program.

[2] [n.d.]. Best Minecraft Plugins in 2020. Retrieved from https://blog.shockbyte.com/best-minecraft-plugins/.

[3] [n.d.]. Group Agreements. Retrieved from http://www.seedsforchange.org.uk/groupagree.

[4] [n.d.]. PolicyKit: Building Governance in Online Communities from Amy Zhang on Vimeo. Retrieved from https: //player.vimeo.com/video/446531759.

[5] [n.d.]. Survival (Game Mode). Retrieved from https://minecraft.fandom.com/wiki/Survival_(Game_Mode).

[6] 2018. Restorative Communities Resource Guide. Retrieved from https://partnershipla.org/wp-content/uploads/2018/ 08/RC-Resource-Guide-2016.pdf.

[7] J. Lawrence Aber, Stephanie M. Jones, Joshua L. Brown, Nina Chaudry, and Faith Samples. 1998. Resolving conflict creatively: Evaluating the developmental effects of a school-based violence prevention program in neighborhood and classroom context. Development and Psychopathology 10, 2 (1998), 187-213.

[8] Yaser Adi, Amanda Killoran, Kulsum Janmohamed, and Sarah Stewart-Brown. 2007. Systematic review of the effectiveness of interventions to promote mental wellbeing in children in primary education. report 1: Universal approaches: non-violence related outcomes. In Database of Abstracts of Reviews of Effects (DARE): Quality-assessed Reviews [Internet]. Centre for Reviews and Dissemination (UK).

[9] June Ahn, Tamara Clegg, Jason Yip, Elizabeth Bonsignore, Daniel Pauw, Lautaro Cabrera, Kenna Hernly, Caroline Pitt, Kelly Mills, Arturo Salazar, Diana Griffing, Jeff Rick, and Rachael Marr. 2018. Science everywhere: Designing public, tangible displays to connect youth learning across settings. In Proceedings of the 2018 CHI Conference on Human Factors in Computing Systems (CHI'18). Association for Computing Machinery, New York, NY, 1-12. DOI : https://doi.org/10.1145/3173574.3173852

[10] Diego Fernando Gutierrez Aponte and Deborah Richards. 2013. Managing cyber-bullying in online educational virtual worlds. In Proceedings of the 9th Australasian Conference on Interactive Entertainment: Matters of Life and Death. ACM Press, New York, New York, 1-9. DOI : https://doi.org/10.1145/2513002.2513006

[11] Flávio S. Azevedo. 2011. Lines of practice: A practice-centered theory of interest relationships. Cognition and Instruction 29, 2 (2011), 147-184.

[12] Flávio S. Azevedo. 2013. The tailored practice of hobbies and its implication for the design of interest-driven learning environments. Fournal of the Learning Sciences 22, 3 (2013), 462-510.

[13] Tom Baranowski, Richard Buday, Debbe I. Thompson, and Janice Baranowski. 2008. Playing for real: video games and stories for health-related behavior change. American fournal of Preventive Medicine 34, 1 (2008), 74-82.

[14] Brigid Barron and Linda Darling-Hammond. 2008. Powerful learning: Studies show deep understanding derives from collaborative methods. Edutopia (October 2008). Retrieved from http://www.edutopia.org/inquiry-project-learningresearch (2008).

[15] Roy F. Baumeister, Ellen Bratslavsky, Catrin Finkenauer, and Kathleen D. Vohs. 2001. Bad is stronger than good. Review of General Psychology 5, 4 (2001), 323-370.

[16] Murtaza H. Baxamusa. 2008. Empowering communities through deliberation the model of community benefits agreements. Journal of Planning Education and Research 27, 3 (2008), 261-276.

[17] Max V. Birk, Benjamin Buttlar, Jason T. Bowey, Susanne Poeller, Shelby C. Thomson, Nicola Baumann, and Regan L. Mandryk. 2016. The effects of social exclusion on play experience and hostile cognitions in digital games. In Proceedings of the 2016 CHI Conference on Human Factors in Computing Systems. ACM, 3007-3019.

[18] Rebecca W. Black. 2008. Adolescents and Online Fan Fiction. Vol. 23. Peter Lang.

[19] Jeremy Blackburn and Haewoon Kwak. 2014. STFU NOOB!. In Proceedings of the 23rd International Conference on World Wide Web. ACM Press, New York, New York, 877-888. DOI : https://doi.org/10.1145/2566486.2567987

[20] Lindsay Blackwell, Jill Dimond, Sarita Schoenebeck, and Cliff Lampe. 2017. Classification and its consequences for online harassment: Design insights from heartMob. Proceedings of the ACM on Human-Computer Interaction 1, CSCW (Dec. 2017), 19 pages. DOI : https://doi.org/10.1145/3134659

[21] Elaine A. Blechman, Ronald J. Prinz, and Jean E. Dumas. 1995. Coping, competence, and aggression prevention: Part 1. Developmental model. Applied and Preventive Psychology 4, 4 (1995), 211-232.

[22] Phyllis C. Blumenfeld, Elliot Soloway, Ronald W. Marx, Joseph S. Krajcik, Mark Guzdial, and Annemarie Palincsar. 1991. Motivating project-based learning: Sustaining the doing, supporting the learning. Educational Psychologist 26, 3-4 (1991), 369-398

[23] Danah Boyd. 2014. It's Complicated: The Social Lives of Networked Teens. Yale University Press.

[24] Virginia Braun and Victoria Clarke. 2012. Thematic analysis. Apa Handbook of Research Methods In Psychology, vol 2. Research Designs: Quantitative, Qualitative, Neuropsychological, and Biological. American Psychological Association, 57-71. 
[25] Amy Bruckman, Pavel Curtis, Cliff Figallo, and Brenda Laurel. 1994. Approaches to managing deviant behavior in virtual communities. In Proceedings of the CHI Conference Companion. 183-184.

[26] Amy S. Bruckman, Jennifer E. Below, Lucas Dixon, Casey Fiesler, Eric E. Gilbert, Sarah A. Gilbert, and J. Nathan Matias. 2018. Managing deviant behavior in online communities III. In Proceedings of the Extended Abstracts of the 2018 CHI Conference on Human Factors in Computing Systems. 1-4.

[27] David Buckingham. 2007. Youth, Identity, and Digital Media. MIT Press.

[28] M. Bundick, M. Andrews, A. Jones, J. M. Mariano, K. C. Bronk, and W. Damon. 2006. Revised youth purpose survey. Stanford Center on Adolescence, Stanford, CA (2006).

[29] Moira Burke, Robert Kraut, and Cameron Marlow. 2011. Social capital on Facebook: Differentiating uses and users. In Proceedings of the SIGCHI Conference on Human Factors in Computing Systems (CHI'11). Association for Computing Machinery, New York, NY, 571-580. DOI : https://doi.org/10.1145/1978942.1979023

[30] Martin Camiré, Pierre Trudel, and Tanya Forneris. 2014. Examining how model youth sport coaches learn to facilitate positive youth development. Physical Education and Sport Pedagogy 19, 1 (2014), 1-17.

[31] Richard F. Catalano, M. Lisa Berglund, Jean A. M. Ryan, Heather S. Lonczak, and J. David Hawkins. 2004. Positive youth development in the United States: Research findings on evaluations of positive youth development programs. The Annals of the American Academy of Political and Social Science 591, 1 (2004), 98-124.

[32] Eshwar Chandrasekharan, Mattia Samory, Shagun Jhaver, Hunter Charvat, Amy Bruckman, Cliff Lampe, Jacob Eisenstein, and Eric Gilbert. 2018. The internet's hidden rules: An empirical study of Reddit norm violations at micro, meso, and macro scales. Proceedings of the ACM on Human-Computer Interaction 2, CSCW (Nov. 2018), 25 pages. DOI : https://doi.org/10.1145/3274301

[33] Suparna Choudhury, Sarah-Jayne Blakemore, and Tony Charman. 2006. Social cognitive development during adolescence. Social Cognitive and Affective Neuroscience 1, 3 (2006), 165-174.

[34] Michael G. Christel, Scott Stevens, Matt Champer, John Balash, Sean Brice, Bryan Maher, Danny Hausmann, Nora Bastida, Chandana Bhargava, and Weiwei Huo. 2013. Beanstalk: A unity game addressing balance principles, socioemotional learning and scientific inquiry. In Proceedings of the 2013 IEEE International Games Innovation Conference (IGIC'13). IEEE, 36-39.

[35] Cathy J. Cohen and Joseph Kahne. 2012. Participatory politics. New media and youth political action. Youth and Participatory Politics Research Network.

[36] Helena Cole and Mark D. Griffiths. 2007. Social interactions in massively multiplayer online role-playing gamers. Cyberpsychology \& Behavior 10, 4 (2007), 575-583.

[37] Margaret Coleman, Larry Wheeler, and Jo Webber. 1993. Research on interpersonal problem-solving training: A review. Remedial and Special Education 14, 2 (1993), 25-37.

[38] National Research Council. 2013. Education for Life and Work: Developing Transferable Knowledge and Skills in the 21st Century. National Academies Press.

[39] Ashley B. Craig, Emily R. Brown, James Upright, and Melissa E. DeRosier. 2016. Enhancing children's social emotional functioning through virtual game-based delivery of social skills training. Journal of Child and Family Studies 25, 3 (2016), 959-968.

[40] John W. Creswell and Cheryl N. Poth. 2016. Qualitative Inquiry and Research Design: Choosing Among Five Approaches. Sage Publications.

[41] Kevin Crowley and Melanie Jacobs. 2002. Building islands of expertise in everyday family activity. In Learning Conversations in Museums. Lawrence Erlbaum Associates Publishers.

[42] William Damon. 2004. What is positive youth development? The Annals of the American Academy of Political and Social Science 591, 1 (2004), 13-24.

[43] William Damon, Jenni Menon, and Kendall Cotton Bronk. 2003. The development of purpose during adolescence. Applied Developmental Science 7, 3 (2003), 119-128.

[44] William DeJong. 1994. Building the Peace: The Resolving Conflict Creatively Program (RCCP). US Department of Justice, Office of Justice Programs, National Institute of Justice.

[45] Ansgar E. Depping and Regan L. Mandryk. 2017. Cooperation and interdependence: How multiplayer games increase social closeness. In Proceedings of the Annual Symposium on Computer-Human Interaction in Play. 449-461.

[46] Nicolas Ducheneaut, Nicholas Yee, Eric Nickell, and Robert J. Moore. 2006. Alone together? exploring the social dynamics of massively multiplayer online games. In Proceedings of the SIGCHI Conference on Human Factors in Computing Systems. ACM, 407-416.

[47] Sean C. Duncan. 2011. Minecraft, beyond construction and survival. Well Played: a fournal on Video Games, Value and Meaning 1, 1 (2011), 1-22.

[48] Joseph A. Durlak. 1998. Common risk and protective factors in successful prevention programs. American fournal of Orthopsychiatry 68, 4 (1998), 512-520. 
[49] Joseph A. Durlak, Roger P. Weissberg, Allison B. Dymnicki, Rebecca D. Taylor, and Kriston B. Schellinger. 2011. The impact of enhancing students' social and emotional learning: A meta-analysis of school-based universal interventions. Child Development 82, 1 (2011), 405-432.

[50] Joseph A. Durlak and Anne M. Wells. 1998. Evaluation of indicated preventive intervention (secondary prevention) mental health programs for children and adolescents. American fournal of Community Psychology 26, 5 (1998), 775802 .

[51] Maurice J. Elias and Harriett Arnold. 2006. The Educator's Guide to Emotional Intelligence and Academic Achievement: Social-Emotional Learning in the Classroom. Corwin Press.

[52] Gisela Ernst. 1994. "Talking circle": Conversation and negotiation in the ESL classroom. Tesol Quarterly 28, 2 (1994), 293-322.

[53] Michael Fielding. 2001. Students as radical agents of change. fournal of Educational Change 2, 2 (2001), $123-141$.

[54] Abbie Reynolds Finlinson, Ann M. Berghout Austin, and Roxane Pfister. 2000. Cooperative games and children's positive behaviors. Early Child Development and Care 164, 1 (2000), 29-40.

[55] Cy Foo and Emi Koivisto. 2004. Defining grief play in MMORPGs: Player and developer perceptions. In Proceedings of the 2004 ACM SIGCHI International Conference on Advances in Computer Entertainment Technology. 245-250. DOI : https://doi.org/10.1145/1067343.1067375

[56] Conrad N. Hilton Foundation. [n.d.]. Supporting Positive Youth Development. Retrieved from https://www. hiltonfoundation.org/news/219-supporting-positive-youth-development.

[57] James Paul Gee. 2003. What video games have to teach us about learning and literacy. Computers in Entertainment 1, 1 (Oct. 2003), 20. DOI : https://doi.org/10.1145/950566.950595

[58] James Paul Gee. 2004. Affinity spaces. In Situated Language and Learning: A Critique of Traditional Schooling. Routledge, 77-89.

[59] James Paul Gee. 2005. Semiotic social spaces and affinity spaces: from The Age of Mythology to today's schools. In Beyond Communities of Practice: Language Power and Social Context, David Barton and Karin Tusting (Eds.). Cambridge University Press, Cambridge, 214-232. DOI : https://doi.org/10.1017/CBO9780511610554.012

[60] James Paul Gee. 2008. Learning and games. In The Ecology of Games: Connecting Youth, Games, and Learning. MIT Press, 21-40.

[61] R. Stuart Geiger and David Ribes. 2011. Trace ethnography: Following coordination through documentary practices. In Proceedings of the 2011 44th Hawaii International Conference on System Sciences. IEEE, 1-10.

[62] Stephen J. Gentles, Cathy Charles, Jenny Ploeg, and K. McKibbon. 2015. Sampling in qualitative research: Insights from an overview of the methods literature. The Qualitative Report 20, 11 (2015), 1772-1789.

[63] Steinunn Gestsdóttir and Richard M. Lerner. 2007. Intentional self-regulation and positive youth development in early adolescence: Findings from the 4-h study of positive youth development.Developmental Psychology 43, 2 (2007), 508.

[64] Shelley Goldman, Angela Booker, and Meghan McDermott. 2008. Mixing the digital, social, and cultural: Learning, identity, and agency in youth participation. Youth, Identity, and Digital Media 216 (2008).

[65] Isabela Granic, Adam Lobel, and Rutger C.M.E. Engels. 2014. The benefits of playing video games. American Psychologist 69, 1 (2014), 66.

[66] Mark T. Greenberg. 2006. Promoting resilience in children and youth: Preventive interventions and their interface with neuroscience. Annals of the New York Academy of Sciences 1094, 1 (2006), 139-150.

[67] Mark T. Greenberg, Celene Domitrovich, and Brian Bumbarger. 2001. The prevention of mental disorders in schoolaged children: Current state of the field.Prevention \& Treatment 4, 1 (2001), 1a.

[68] Mark T. Greenberg, Roger P. Weissberg, Mary Utne O’Brien, Joseph E. Zins, Linda Fredericks, Hank Resnik, and Maurice J. Elias. 2003. Enhancing school-based prevention and youth development through coordinated social, emotional, and academic learning. American Psychologist 58, 6-7 (2003), 466.

[69] James G. Greeno and Yrjö Engeström. 2014. Learning in activity. In The Cambridge Handbook of the Learning Sciences (2nd ed.), R. Keith Sawyer (Ed.). Cambridge University Press, Cambridge, 128-148. DOI : https://doi.org/10.1017/ CBO9781139519526.009

[70] Mark Griffiths, Zaheer Hussain, Sabine M. Grüsser, Ralf Thalemann, Helena Cole, Mark NO Davies, and Darren Chappell. 2011. Social interactions in online gaming. International fournal of Game-Based Learning 1, 4 (2011), 2036.

[71] James Grimmelmann. 2015. The Virtues of Moderation. Yale Journal of Law and Technology 17, 1 (September 2015) Retrieved from https://digitalcommons.law.yale.edu/yjolt/vol17/iss1/2

[72] Nancy G. Guerra and Catherine P. Bradshaw. 2008. Linking the prevention of problem behaviors and positive youth development: Core competencies for positive youth development and risk prevention. New Directions for Child and Adolescent Development 2008, 122 (2008), 1-17.

[73] Julia Katherine Haines. 2017. Towards multi-dimensional ethnography. In Ethnographic Praxis in Industry Conference Proceedings, Vol. 2017. Wiley Online Library, 127-141. 
[74] Robert Halpern. 2002. A different kind of child development institution: The history of after-school programs for low-income children. Teachers College Record 104, 2 (2002), 178-211.

[75] Eszter Hargittai. 2002. Beyond logs and surveys: In-depth measures of people's web use skills. fournal of the American Society for Information Science and Technology 53, 14 (2002), 1239-1244.

[76] Lisa Harker, Sonja Jütte, Tom Murphy, Holly Bentley, Pam Miller, and Kate Fitch. 2013. How safe are our children. London: National Society for the Prevention of Cruelty to Children.

[77] Alexa M. Harris, Diego Gómez-Zará, Leslie A. DeChurch, and Noshir S. Contractor. 2019. Joining together online: the trajectory of CSCW scholarship on group formation. Proceedings of the ACM on Human-Computer Interaction 3 , CSCW (Nov. 2019), 27 pages. DOI : https://doi.org/10.1145/3359250

[78] Uwe Hasebrink, Anke Görzig, Leslie Haddon, Veronika Kalmus, and Sonia Livingstone. 2011. Patterns of risk and safety online: In-depth analyses from the EU Kids Online survey of 9- to 16-year-olds and their parents in 25 European countries. EU Kids Online

[79] Uwe Hasebrink, Sonia Livingstone, Leslie Haddon, and Kjartan Olafsson. 2009. Comparing children's online opportunities and risks across Europe: Cross-national comparisons for EU Kids Online. EU Kids Online.

[80] Carrie Heeter. 1992. Being there: The subjective experience of presence. Presence: Teleoperators \& Virtual Environments 1, 2 (1992), 262-271.

[81] Ellen Johanna Helsper. 2012. A corresponding fields model for the links between social and digital exclusion. Communication Theory 22, 4 (2012), 403-426.

[82] Roberta Anna Heydenberk and Warren R. Heydenberk. 2005. Increasing meta-cognitive competence through conflict resolution. Education and Urban Society 37, 4 (2005), 431-452.

[83] Mary Helen Immordino-Yang, Linda Darling-Hammond, and Christina Krone. 2018. The brain basis for integrated social, emotional, and academic development. Washington, DC: National Commission on Social, Emotional, and Academic Development.

[84] Mizuko Ito, Sonja Baumer, Matteo Bittanti, and Rachel Cody. 2019. Hanging Out, Messing Around, and Geeking Out: Kids Living and Learning with New Media. MIT Press.

[85] Mizuko Ito, Kris Gutiérrez, Sonia Livingstone, Bill Penuel, Jean Rhodes, Katie Salen, Juliet Schor, Julian Sefton-Green, and S. Craig Watkins. 2013. Connected Learning: An Agenda for Research and Design. Digital Media and Learning Research Hub.

[86] Mizuko Ito, Crystle Martin, Rachel Cody Pfister, Matthew H. Rafalow, Katie Salen, and Amanda Wortman. 2018. Affinity Online: How Connection and Shared Interest Fuel Learning. Vol. 2. NYU Press.

[87] David W. Johnson and Roger T. Johnson. 1995. Reducing School Violence Through Conflict Resolution. ASCD.

[88] David W. Johnson and Roger T. Johnson. 1995. Teaching students to be peacemakers: Results of five years of research. Peace and Conflict 1, 4 (1995), 417-438.

[89] David W. Johnson and Roger T. Johnson. 1996. Reducing school violence through conflict resolution training. Nassp Bulletin 80, 579 (1996), 11-18.

[90] Stephanie M. Jones, Joshua L. Brown, and J. Lawrence Aber. 2010. Three year cumulative impacts of the 4Rs program on children's social-emotional, behavioral, and academic outcomes.Society for Research on Educational Effectiveness.

[91] Tricia S. Jones. 2004. Conflict resolution education: The field, the findings, and the future. Conflict Resolution Quarterly 22, 1-2 (2004), 233

[92] Yasmin B. Kafai. 2010. World of Whyville: An introduction to tween virtual life. Games and Culture 5, 1 (2010), 3-22.

[93] Joseph Kahne, Ellen Middaugh, Nam-Jin Lee, and Jessica T. Feezell. 2012. Youth online activity and exposure to diverse perspectives. New Media \& Society 14, 3 (2012), 492-512.

[94] Mark E. Kann, Jeff Berry, Connor Grant, and Phil Zager. 2007. The internet and youth political participation. First Monday 12, 8 (2007).

[95] Beeban Kidron and Angharad Rudkin. 2017. Digital childhood: Addressing childhood development milestones in the digital environment. Project Report, 5Rights Foundation. Retrieved May 20, 2021 from https://eprints.soton.ac. uk/416911/.

[96] Douglas Kirby and Karin Coyle. 1997. Youth development programs. Children and Youth Services Review 19, 5-6 (1997), 437-454.

[97] Neta Kligler-Vilenchik and Ioana Literat. 2018. Distributed creativity as political expression: Youth responses to the 2016 US presidential election in online affinity networks. Fournal of Communication 68, 1 (2018), 75-97.

[98] Yubo Kou and Bonnie A. Nardi. 2014. Governance in League of Legends: A hybrid system. In Proceedings of the International Conference on the Foundations of Digital Games.

[99] Yong Ming Kow and Timothy Young. 2013. Media technologies and learning in the starcraft esport community. In Proceedings of the 2013 Conference on Computer Supported Cooperative Work. 387-398.

[100] Haewoon Kwak and Jeremy Blackburn. 2014. Linguistic analysis of toxic behavior in an online video game. In Proceedings of the International Conference on Social Informatics. Springer, 209-217. 
[101] Haewoon Kwak, Jeremy Blackburn, and Seungyeop Han. 2015. Exploring cyberbullying and other toxic behavior in team competition online games. In Proceedings of the 33rd Annual ACM Conference on Human Factors in Computing Systems. ACM, 3739-3748.

[102] Haewoon Kwak, Jeremy Blackburn, and Seungyeop Han. 2015. Exploring cyberbullying and other toxic behavior in team competition online games. In Proceedings of the 33rd Annual ACM Conference on Human Factors in Computing Systems. ACM Press, New York, New York, 3739-3748. DOI : https://doi.org/10.1145/2702123.2702529

[103] Cliff Lampe, Paul Zube, Jusil Lee, Chul Hyun Park, and Erik Johnston. 2014. Crowdsourcing civility: A natural experiment examining the effects of distributed moderation in online forums. Government Information Quarterly 31 , 2 (2014), 317-326.

[104] Reed W. Larson. 2000. Toward a psychology of positive youth development.American Psychologist 55, 1 (2000), 170.

[105] Jean Lave. 1991. Situating learning in communities of practice. In Perspectives on Socially Shared Cognition. American Psychological Association, Washington, DC, US, 63-82. DOI : https://doi.org/10.1037/10096-003

[106] Kevin M. Leander and Kelly K. McKim. 2003. Tracing the everyday 'sitings' of adolescents on the internet: A strategic adaptation of ethnography across online and offline spaces. Education, Communication \& Information 3, 2 (2003), 211-240.

[107] Amanda Lenhart, Mary Madden, Aaron Smith, Kristen Purcell, Kathryn Zickuhr, and Lee Rainie. 2011. Teens, kindness and cruelty on social network sites: How American teens navigate the new world of "Digital Citizenship". Pew Internet \& American Life Project.

[108] Richard M. Lerner, Cornelia Brentano, Elizabeth M. Dowling, and Pamela M. Anderson. 2002. Positive youth development: Thriving as the basis of personhood and civil society. New Directions for Youth Development 2002, 95 (2002), $11-34$.

[109] Richard M. Lerner, Jacqueline V. Lerner, Jason B. Almerigi, Christina Theokas, Erin Phelps, Steinunn Gestsdottir, Sophie Naudeau, Helena Jelicic, Amy Alberts, and Lang Ma. 2005. Positive youth development, participation in community youth development programs, and community contributions of fifth-grade adolescents: Findings from the first wave of the 4-H study of positive youth development. The fournal of Early Adolescence 25, 1 (2005), 17-71.

[110] Richard M. Lerner, Jacqueline V. Lerner, and Janette B. Benson. 2011. Positive youth development: Research and applications for promoting thriving in adolescence. In Advances in Child Development and Behavior. Vol. 41. Elsevier, $1-17$.

[111] Junlei Li and Megan M. Julian. 2012. Developmental relationships as the active ingredient: A unifying working hypothesis of "what works" across intervention settings. American fournal of Orthopsychiatry 82, 2 (2012), 157.

[112] Eden Litt. 2013. Measuring users' internet skills: A review of past assessments and a look toward the future. New Media \& Society 15, 4 (2013), 612-630.

[113] Sonia Livingstone. 2013. Online risk, harm and vulnerability: Reflections on the evidence base for child Internet safety policy. ZER: fournal of Communication Studies 18, 35 (2013), 13-28.

[114] Sonia Livingstone. 2016. Reframing media effects in terms of children's rights in the digital age. Fournal of Children and Media 10, 1 (2016), 4-12.

[115] Sonia Livingstone, Leslie Haddon, Anke Görzig, and Kjartan Olafsson. 2011. Risks and safety on the internet: the perspective of European children: full findings and policy implications from the EU Kids Online survey of 9-16 year olds and their parents in 25 countries. EU Kids Online.

[116] Mechthild Maczewski. 2002. Exploring identities through the internet: Youth experiences online. In Child and Youth Care Forum, Vol. 31. Springer, 111-129.

[117] Brendan Maher. 2016. Can a video game company tame toxic behaviour? Nature News 531, 7596 (2016), 568.

[118] Brendan Maher. 2016. Good gaming: scientists are helping to tame toxic behaviour in the worlds most popular online game. Nature 531, 7596 (2016), 568-572.

[119] Jenni Menon Mariano and Julie Going. 2011. Youth purpose and positive youth development. In Advances in Child Development and Behavior. Vol. 41. Elsevier, 39-68.

[120] Adrienne Massanari. 2015. \#Gamergate and The Fappening: How reddit's algorithm, governance, and culture support toxic technocultures. New Media \& Society 19, 3 (2015), 329-346. DOI : https://doi.org/10.1177/1461444815608807

[121] J. Nathan Matias. 2019. Preventing harassment and increasing group participation through social norms in 2,190 online science discussions. Proceedings of the National Academy of Sciences 116, 20 (2019), 9785-9789.

[122] Gregor McEwan, Carl Gutwin, Regan L. Mandryk, and Lennart Nacke. 2012. "I'm Just Here to play games": social dynamics and sociality in an online game site. In Proceedings of the ACM 2012 Conference on Computer Supported Cooperative Work (CSCW'12). Association for Computing Machinery, New York, NY, 549-558. DOI : https://doi.org/ $10.1145 / 2145204.2145289$

[123] Kristen L. Merrill, Stephen W. Smith, Michelle M. Cumming, and Ann P. Daunic. 2017. A review of social problemsolving interventions: Past findings, current status, and future directions. Review of Educational Research 87, 1 (2017), 71-102. 
[124] Dana L. Mitra. 2004. The significance of students: can increasing "student voice" in schools lead to gains in youth development? Teachers College Record 106 (2004), 651-688. DOI : https://doi.org/10.1111/j.1467-9620.2004.00354.x

[125] Dana L. Mitra. 2008. Amplifying student voice. Educational Leadership 66, 3 (2008), 20-25.

[126] Kathryn Montgomery. 2000. Youth and digital media: A policy research agenda. fournal of Adolescent Health 27, 2 (2000), 61-68.

[127] Kathryn C. Montgomery, Jennifer Earl, Kirsten A. Foot, and Michael Xenos. 2008. Civic Life Online: Learning How Digital Media Can Engage Youth. Mit Press.

[128] Nick Oatley and Rashmi Thapa. 2012. Media, youth and conflict prevention in sierra leone. Retrieved from http: //www.ifp-ew.eu/pdf/201204IfPEWMediaYouthConflictPreventionSalone.pdf (2012).

[129] Ray Oldenburg. 1999. The Great Good Place: Cafes, Coffee Shops, Bookstores, Bars, Hair Salons, and Other Hangouts at the Heart of a Community. Da Capo Press.

[130] Judith S. Olson and Wendy A. Kellogg. 2014. Ways of Knowing in HCI. Vol. 2. Springer.

[131] National Scientific Council on the Developing Child. 2007. The Timing and Quality of Early Experiences Combine to Shape Brain Architecture: Working Paper \#5. Retrieved from http://www.developingchild.net.

[132] Elinor Ostrom. 2000. Collective action and the evolution of social norms. Fournal of Economic Perspectives 14, 3 (2000), 137-158.

[133] John G. Palfrey, Urs Gasser, Miriam Simun, and Rosalie Barnes. 2009. Youth, creativity, and copyright in the digital age. Berkman Center Research Publication 2009-05.

[134] Elizabeth Levy Paluck. 2009. What's in a norm? Sources and processes of norm change. fournal of Personality and Social Psychology 96, 3 (2009), 594-600.

[135] Elizabeth Levy Paluck and Hana Shepherd. 2012. The salience of social referents: A field experiment on collective norms and harassment behavior in a school social network. Journal of Personality and Social Psychology 103, 6 (2012), 899.

[136] Kylie A. Peppler and Yasmin B. Kafai. 2007. Collaboration, computation, and creativity: media arts practices in urban youth culture. In Proceedings of the 8th International Conference on Computer Supported Collaborative Learning. International Society of the Learning Sciences, 590-592.

[137] H. Wesley Perkins. 2002. Social norms and the prevention of alcohol misuse in collegiate contexts. Fournal of Studies on Alcohol, Supplement 14 (2002), 164-172.

[138] Karen J. Pittman and Wanda E. Fleming. 1991. Academy for Educational Development, United States, Congress, House, and Youth Select Committee on Children and Families. A new vision: promoting youth development: testimony of Karen J. Pittman before the House Select Committee on Children, Youth and Families. Project Report. Academy for Educational Development, Washington, DC.

[139] Karen Pittman, Merita Irby, and Thaddeus Ferber. 2001. Unfinished business: Further reflections on a decade of promoting youth development. In Trends in Youth Development. Springer, 3-50.

[140] Karen Johnson Pittman, Merita Irby, Joel Tolman, Nicole Yohalem, and Thaddeus Ferber. 2011. Preventing problems, promoting development, encouraging engagement. Forum for Youth Investment.

[141] Privacy Policy and Terms of Service. 2017. Positive Youth Development: Research, Principles, and Practices. Retrieved from https:/www.rootsofaction.com/positive-youth-development/.

[142] Nathaniel Poor and Marko M. Skoric. 2014. Death of a guild, birth of a network: Online community ties within and beyond code. Games and Culture 9, 3 (2014), 182-202.

[143] Andrew K. Przybylski, C. Scott Rigby, and Richard M. Ryan. 2010. A motivational model of video game engagement. Review of General Psychology 14, 2 (2010), 154-166.

[144] C. Cybele Raver. 2002. Emotions matter: Making the case for the role of young children's emotional development for early school readiness. Social Policy Report 16, 3 (2002), 1-20.

[145] Paul Resnick. 2001. Beyond bowling together: Sociotechnical capital. HCI in the New Millennium 77 (2001), 247-272.

[146] Kathryn E. Ringland. 2019. "Autsome": Fostering an autistic identity in an online Minecraft community for youth with autism. In Proceedings of the 14th International Conference on Information in Contemporary Society. 132-143. DOI : 10.1007/978-3-030-15742-5_12

[147] Kathryn E. Ringland, Christine T. Wolf, Lynn Dombrowski, and Gillian R. Hayes. 2015. Making safe: Communitycentered practices in a virtual world dedicated to children with autism. In Proceedings of the 18th ACM Conference on Computer Supported Cooperative Work \& Social Computing. ACM, 1788-1800.

[148] Kathryn E. Ringland, Christine T. Wolf, Heather Faucett, Lynn Dombrowski, and Gillian R. Hayes. 2016. "Will I always be not social?": Re-conceptualizing sociality in the context of a Minecraft community for autism. In Proceedings of the 2016 CHI Conference on Human Factors in Computing Systems. ACM Press, Santa Clara, California, 1256-1269. DOI : https://doi.org/10.1145/2858036.2858038

[149] Jane Ritchie, Jane Lewis, and Gillian Elam. 2003. Designing and Selecting Samples. London: Sage. 
[150] Barbara Rogoff. 1994. Developing understanding of the idea of communities of learners. Mind, Culture, and Activity 1, 4 (1994), 209-229.

[151] Barbara Rogoff, Jacqueline Baker-Sennett, Pilar Lacasa, and Denise Goldsmith. 1995. Development through participation in sociocultural activity. New Directions for Child and Adolescent Development 1995, 67 (1995), 45-65.

[152] Louis Rolfes and Kathrin Passig. 2019. The proto-governance of minecraft servers. fournal For Virtual Worlds Research 12, 3 (2019), 1-9.

[153] Kenneth H. Rubin and Linda Rose-Krasnor. 1992. Interpersonal problem solving and social competence in children. In Handbook of Social Development. Springer, 283-323.

[154] Stephen P. Safran and Karen Oswald. 2003. Positive behavior supports: Can schools reshape disciplinary practices? Exceptional Children 69, 3 (2003), 361-373.

[155] Katie Salen. 2008. Toward an ecology of gaming. In The Ecology of Games: Connecting Youth, Games, and Learning. The MIT Press, 1-20. DOI : 10.1162/dmal.9780262693646.001

[156] Mizuko Ito, Richard Arum, Dalton Conley, Kris Gutiérrez, Ben Kirshner, Sonia Livingstone, Vera Michalchik, William Penuel, Kylie Peppler, Nichole Pinkard, Jean Rhodes, Katie Salen Tekinbaş, Juliet Schor, Julian Sefton-Green, and S. Craig Watkins. 2020. The Connected Learning Research Network: Reflections on a Decade of Engaged Scholarship. Connected Learning Alliance, Irvine, CA.

[157] Katie Salen and Katie Salen Tekinba? 2008. TThe Ecology of Games: Connecting Youth, Games, and Learning. MIT Press.

[158] Joseph Seering, Robert Kraut, and Laura Dabbish. 2017. Shaping pro and anti-social behavior on twitch through moderation and example-setting. In Proceedings of the 2017 ACM Conference on Computer Supported Cooperative Work and Social Computing. ACM Press, Portland, Oregon, 111-125. DOI : https://doi.org/10.1145/2998181.2998277

[159] Joseph Seering, Tony Wang, Jina Yoon, and Geoff Kaufman. 2019. Moderator engagement and community development in the age of algorithms. New Media \& Society 21, 7 (July 2019), 1417-1443. DOI : https://doi.org/10.1177/ 1461444818821316

[160] Martin E. P. Seligman. 2002. Positive psychology, positive prevention, and positive therapy. Handbook of Positive Psychology 2, 2002 (2002), 3-12.

[161] Martin E. P. Seligman. 2007. Coaching and positive psychology. Australian Psychologist 42, 4 (2007), $266-267$.

[162] Martin E. P. Seligman and Mihaly Csikszentmihalyi. 2014. Positive psychology: An introduction. In Flow and the Foundations of Positive Psychology. Springer, 279-298.

[163] Eva Sharma and Munmun De Choudhury. 2018. Mental health support and its relationship to linguistic accommodation in online communities. In Proceedings of the 2018 CHI Conference on Human Factors in Computing Systems (CHI'18). Association for Computing Machinery, New York, NY, 1-13. DOI : https://doi.org/10.1145/3173574.3174215

[164] Myrna B. Shure. 2000. I Can Problem Solve: Intermediate Elementary Grades. Vol. 3. Research Press.

[165] Myrna B. Shure. 2001. I can problem solve (ICPS): An interpersonal cognitive problem solving program for children. Residential Treatment for Children \& Youth 18, 3 (2001), 3-14

[166] Myrna B. Shure and Bonnie Aberson. 2005. Enhancing the process of resilience through effective thinking. In Handbook of Resilience in Children. Springer, 373-394.

[167] Myrna B. Shure and Roberta Israeloff. 2001. Raising a Thinking Preteen: The "I Can Problem Solve" Program for 8- to 12-Year-Olds. Macmillan.

[168] Myrna B. Shure and George Spivack. 1980. Interpersonal problem solving as a mediator of behavioral adjustment in preschool and kindergarten children. fournal of Applied Developmental Psychology 1, 1 (1980), 29-44.

[169] Myrna B. Shure and George Spivack. 1982. Interpersonal problem-solving in young children: A cognitive approach to prevention. American Journal of Community Psychology 10, 3 (1982), 341-356.

[170] Petr Slovak, Katie Salen, Stephanie Ta, and Geraldine Fitzpatrick. 2018. Mediating conflicts in minecraft: Empowering learning in online multiplayer games. In Proceedings of the 2018 CHI Conference on Human Factors in Computing Systems (CHI'18). ACM, New York, NY, 595:1-595:13. DOI : https://doi.org/10.1145/3173574.3174169

[171] Petr Slovák and Geraldine Fitzpatrick. 2015. Teaching and developing social and emotional skills with technology. ACM Transactions on Computer-Human Interaction 22, 4 (2015), 19.

[172] Petr Slovák, Nikki Theofanopoulou, Alessia Cecchet, Peter Cottrell, Ferran Altarriba Bertran, Ella Dagan, Julian Childs, and Katherine Isbister. 2018. I just let him cry...: Designing socio-technical interventions in families to prevent mental health disorders. Proceedings of the ACM on Human-Computer Interaction 2, CSCW (2018), 160.

[173] Petr Slovák, Greg Wadley, David Coyle, Anja Thieme, Naomi Yamashita, Reeva Lederman, and Mia Doces. 2015. Developing skills for social and emotional wellbeing. In Proceedings of the 33rd Annual ACM Conference Extended Abstracts on Human Factors in Computing Systems. ACM, 2397-2400.

[174] Jonas Heide Smith. 2004. Playing dirty - understanding conflicts in multiplayer games. In Proceedings of the 5th Annual Conference of The Association of Internet Researchers. 19-22. 
[175] George Spivack and Myrna B. Shure. 1989. Interpersonal cognitive problem solving (ICPS): A competence-building primary prevention program. Journal of Prevention \& Intervention in the Community 6, 2 (1989), 151-178.

[176] Kurt Squire. 2013. Video games and learning: Teaching and participatory culture in the digital age. Alberta fournal of Educational Research 59, 1 (2013), 129-132.

[177] Constance A. Steinkuehler. 2004. Learning in massively multiplayer online games. In Proceedings of the 6th International Conference on Learning Sciences. International Society of the Learning Sciences, 521-528.

[178] R. S. Stern, T. B. Harding, A. A. Holzer, and N. A. Elbertson. 2015. Current and potential uses of technology to enhance SEL. What's now and what's next. In Handbook of Social and Emotional Learning. Research and Practice. Guilford Publications, 516-531.

[179] Janet Sternberg. 2012. Misbehavior in Cyber Places: The Regulation of Online Conduct in Virtual Communities on the Internet. Rowman \& Littlefield.

[180] Hari Sundaram, Yu-Ru Lin, Munmun De Choudhury, and Aisling Kelliher. 2012. Understanding community dynamics in online social networks: a multidisciplinary review. IEEE Signal Processing Magazine 29, 2 (2012), 33-40.

[181] Robert H. Tai, Philip M. Sadler, and Adam V. Maltese. 2007. A study of the association of autonomy and achievement on performance. 16, 1 (2007), 7 .

[182] Margaret E. Tankard and Elizabeth Levy Paluck. 2016. Norm perception as a vehicle for social change. Social Issues and Policy Review 10, 1 (2016), 181-211.

[183] Rebecca D. Taylor, Eva Oberle, Joseph A. Durlak, and Roger P. Weissberg. 2017. Promoting positive youth development through school-based social and emotional learning interventions: A meta-analysis of follow-up effects. Child Development 88, 4 (2017), 1156-1171.

[184] Tina L. Taylor. 2006. Beyond management: Considering participatory design and governance in player culture. First Monday (2006).

[185] Barbara Tedlock. 1991. From participant observation to the observation of participation: The emergence of narrative ethnography. Journal of Anthropological Research 47, 1 (1991), 69-94.

[186] Tracy. 2015. Setting Group Agreements with Youth. Retrieved from https://heartmindonline.org/resources/settinggroup-agreements-with-youth.

[187] John A. Velez. 2015. Extending the theory of bounded generalized reciprocity: An explanation of the social benefits of cooperative video game play. Computers in Human Behavior 48 (2015), 481-491. DOI : https://doi.org/10.1016/j. chb.2015.02.015

[188] John A. Velez and David R. Ewoldsen. 2013. Helping behaviors during video game play. Fournal of Media Psychology 25, 4 (2013), 190-200.

[189] Jessica Vitak, Kalyani Chadha, Linda Steiner, and Zahra Ashktorab. 2017. Identifying women's experiences with and strategies for mitigating negative effects of online harassment. In Proceedings of the 2017 ACM Conference on Computer Supported Cooperative Work and Social Computing. 1231-1245.

[190] Katherine Weare and Melanie Nind. 2011. Mental health promotion and problem prevention in schools: what does the evidence say? Health Promotion International 26, suppl_1 (2011), i29-i69.

[191] Carolyn Webster-Stratton and M. Jamila Reid. 2004. Strengthening social and emotional competence in young children-The foundation for early school readiness and success: Incredible years classroom social skills and problem-s curriculum. Infants \& Young Children 17, 2 (2004), 96-113.

[192] Roger P. Weissberg and Mary Utne O’Brien. 2004. What works in school-based social and emotional learning programs for positive youth development. The Annals of the American Academy of Political and Social Science 591, 1 (2004), 86-97.

[193] Etienne Wenger. 1998. Communities of practice: Learning as a social system. Systems thinker 9, 5 (1998), 2-3.

[194] Etienne Wenger. 2011. Communities of practice: A brief introduction. (2011).

[195] Paulette Running Wolf and Julie A. Rickard. 2003. Talking circles: A native American approach to experiential learning. Fournal of Multicultural Counseling and Development 31, 1 (2003), 39-43. DOI : https://doi.org/10.1002/j. 2161-1912.2003.tb00529.x

[196] Michelle F. Wright and Yan Li. 2011. The associations between young adults' face-to-face prosocial behaviors and their online prosocial behaviors. Computers in Human Behavior 27, 5 (2011), 1959-1962.

[197] Nick Yee. 2006. Motivations for play in online games. CyberPsychology \& Behavior 9, 6 (2006), 772-775.

[198] Joseph E. Zins. 2004. Building Academic Success on Social and Emotional Learning: What Does the Research Say? Teachers College Press.

Received December 2019; revised September 2020; accepted February 2021 